\title{
The Face of Hypervirulent Klebsiella Pneumoniae (hvKp) Isolated from Clinical Samples of Two Iranian Teaching Hospitals
}

\author{
Rahimeh Sanikhani \\ Pasteur Institute of Iran \\ Mohammad Moeinirad \\ Tehran University of Medical Sciences \\ Hamid Solgi \\ Isfahan University of Medical Sciences \\ Azar Haddadi \\ Tehran University of Medical Sciences \\ Fereshteh Shahcheraghi \\ Pasteur Institute of Iran \\ Farzad Badmasti ( $\sim$ fbadmasti2008@gmail.com ) \\ Pasteur Institute of Iran
}

\section{Research}

Keywords: Hypervirulent Klebsiella pneumonia, Identification test, Antimicrobial resistance, Clonal relatedness

Posted Date: June 11th, 2021

DOI: https://doi.org/10.21203/rs.3.rs-589877/v1

License: (c) (i) This work is licensed under a Creative Commons Attribution 4.0 International License. Read Full License 


\begin{abstract}
Hypervirulent Klebsiella pneumoniae (hvKp) has emerged as a pathogen of global concern. In this study, both phenotypic and genotypic tests were used to detect hvKp. Antimicrobial resistance profiles and clonal relatedness of clinical isolates were also determined. We found that $62.6 \%$ of the isolates were tellurite resistant and among them iucA or iutA or peg344 as hvKp molecular markers, were positive. The bla $a_{\mathrm{SHV}}(81.4 \%)$, followed by bla $a_{\mathrm{CTX}-\mathrm{M} 15}(75.5 \%)$ and $b / a_{\mathrm{TEM}}(67.6 \%), b / a_{0 X \mathrm{X}-48}(33.7 \%)$, bla $a_{\mathrm{NDM}-1}(32.3 \%)$ were detected, while bla $\mathrm{KPC}-1_{1}$ was not present in any hvKp isolates. It was found that the majority of hvKp isolates belonged to capsular serotype $\mathrm{K} 20$ and ompK36 group C, which is related to CG23 (e.g. ST23). A high percentage of multidrug-resistant hvKp (MDR-hvKp) and high resistance to imipenem (66\%) indicated that there is an urgent problem that should be addressed in the clinical settings.
\end{abstract}

\title{
1. Introduction
}

Hypervirulent Klebsiella pneumoniae (hvKp), an emerging pathotype of K. pneumoniae was first reported from Taiwan. It was identified as an important cause of pyogenic liver abscess $(1,2)$. In hvKp isolates, pLVPK-like plasmids (Large Virulence Plasmid of K. pneumoniae) encoding virulence factor genes including capsular polysaccharide synthesis regulators ( $r m p A$ and $r m p A 2)$ and iron acquisition systems (iuc, iut, and iro siderophore gene cluster), a metabolic transporter (peg-344) and also heavy metal resistance genes (copper, silver, lead, and tellurite), have been identified (3). Therefore, most hvKp isolates are able to reduce tellurite and form a black colony due to the presence of the major virulence plasmids containing a tellurite resistance gene (4). The pLVPK-like plasmids may carry all virulence factor genes or have lost some of them $(5,6)$. On the other hand, acquisition of antibiotic resistance plasmids or insertion of resistant mobile genetic elements into the hvKp plasmid turns them into superbugs that can be termed hyper-resistant hvKp strains (7-9). Some K. pneumoniae clones are characterized as high-risk clones that play an important role in the spread of antibiotic-resistant strains $(10,11)$.

The association of the porin ompK36 with clonal relatedness of $K$. pneumoniae isolates has been described in several studies $(12,13)$. Four different genotypes for ompK36 porin (A to D) in K. pneumoniae were defined and the correlation of different variants of ompK36 with specific sequence types (STs) was illustrated $(10,12,13)$. The objectives of the current study were to investigate the phenotypic and genotypic identification of hvKp isolates, prevalence of virulence factors, antibiotic resistance and molecular epidemiology of hvKp strains obtained from two Iranian educational hospitals.

\section{Materials And Methods}

\subsection{Bacterial isolation and identification}

In this cross-sectional study, we collected a total of 477 non-repetitive $K$. pneumoniae as clinical isolates from two educational hospitals in Tehran over a period of time from June 2019 to December 2020. All bacterial isolates were identified using standard biochemical laboratory methods and then the isolates were stored in a freezer at $-70^{\circ} \mathrm{C}$ in nutrient broth containing $20 \%$ glycerol until further studies.

\subsection{HvKp phenotypic identification}

\subsubsection{Tellurite resistance}

We used tellurite agar culture as a rapid screening test in this study. The isolates that formed black colonies on this telluritecontaining selective medium were selected as presumptive hypervirulent strains for further study. For this purpose, $0.1 \mathrm{~g}$ of potassium tellurite powder was first dissolved in $10 \mathrm{ml}$ of sterile distilled water and filtered using membrane filters of pore size $0.45 \mu \mathrm{m}$. Then we added $300 \mu \mathrm{l}$ of the potassium tellurite solution to $100 \mathrm{ml}$ of Mueller-Hinton agar medium, which was autoclaved and cooled to $45-50^{\circ} \mathrm{C}$. Finally, we dispensed into sterile plates. Colonies were examined after overnight incubation at $37^{\circ} \mathrm{C}$ (This study).

\subsubsection{String Test}


Hypermucoviscous phenotype of the hvKp isolates was examined by the string test, and the positive test was confirmed via the formation of a 5 -mm mucoviscous filament by stretching of bacterial colonies on a blood agar after $24 \mathrm{~h}$ of incubation at $37^{\circ} \mathrm{C}$ (14).

\subsection{Molecular Characteristics}

\subsubsection{DNA extraction and identification}

Plasmid DNA extraction Mini Kit (FAVORGEN Biotech Corporation, Taiwan) has been used for the detection of genes carried on plasmids. In addition, the boiling method was used for isolation of genomic DNA (15). All amplification reactions for PCR assays were prepared in a total volume of $25 \mu$ l. The list of primer sequences, PCR product sizes, and PCR conditions is shown in Table 1. Finally, all PCR amplification products were sequenced and then matched against the GenBank database using BLAST tool (http://www.ncbi.nlm.nih.gov/blast/).

\subsubsection{HvKp molecular identification}

All tellurite-resistant K. pneumoniae were screened for the presence of the aerobactin (iucA), its receptor (iutA) genes and peg344. The isolates containing the iucA or iutA or peg344 genes were considered as hvKps (16).

\subsection{Antimicrobial Susceptibility testing}

Antimicrobial susceptibility testing was performed using the disc diffusion

method. according to the clinical and laboratory standards institute (CLSI) guidelines (CLSI 2018-M100-S28) by the following antibiotic discs including amikacin (AK), gentamicin (GN), cefotaxime (CTX), ceftazidime (CAZ), ceftriaxone (CRO), imipenem (IMI), meropenem (MRP), cefepime (FEP), ciprofloxacin (CIP), ampicillin (AMP) and aztreonam (AZM). Minimum inhibitory concentration (MICs) of imipenem and ceftazidime were determined by broth dilution method. Escherichia coli ATCC 25922 was used as the quality control strain for antimicrobial susceptibility testing.

\subsection{Capsular genotyping and detection of virulence genes, and antimicrobial resistance genes}

The hvKp capsular serotypes K1, K2, K5, K20, K54, and K57 were identified using PCR method. The hvKp virulence genes including salmochelin siderophore (iroB), mucoviscosity-associated gene ( $m a g A)$, Klebsiella ferric uptake ( $k f u)$, yersiniabactin $(y b t)$, allantoin metabolism gene (allS), and $r m p A$ were detected by specific primers listed in Table 1. In addition, PCR assays were carried out for detection of $b / a_{\mathrm{TEM}}$, bla $a_{\mathrm{SHV}}$, and $b / a_{\mathrm{CTX}-\mathrm{M} 15}, b / a_{\mathrm{KPC}-1}, b / a_{\mathrm{NDM}-1}$, and $b / a_{\mathrm{OXA}-48}$ genes for all hvKp isolates.

\subsection{Determination of clonal relatedness using ompK36 typing}

All hvKp isolates were subjected to ompK36 typing by the PCR-based method described by Yan et al., using four primer pairs (12). 
Primers, product size, and annealing temperature used for this study

\begin{tabular}{|c|c|c|c|c|}
\hline Primer name & Primer sequence $\left(5^{\prime} \rightarrow 3^{\prime}\right)$ & $\begin{array}{l}\text { Amplicon } \\
\text { size (bp) }\end{array}$ & $\begin{array}{l}\text { Annealing } \\
\text { temperature }\left({ }^{\circ} \mathrm{C}\right)\end{array}$ & REF. \\
\hline \multirow[t]{2}{*}{ iucA } & F: AATCAATGGCTATTCCCGCTG & 239 & 62 & $(16)$ \\
\hline & R: CGCTTCACTTCTTTCACTGACAGG & & & \\
\hline \multirow[t]{2}{*}{ peg 344} & F: GCGGGAAAGGACAGAAAGCCAGTG & 332 & 56 & This study \\
\hline & R: GAGGGAAGATGAGAAATACGAGC & & & \\
\hline \multirow[t]{2}{*}{ iutA } & F: GCCGCTAGGTTGGTGATGT & 949 & 61 & This study \\
\hline & R: CTCTGGTCGTGCTGGTTGA & & & \\
\hline \multirow[t]{2}{*}{ iroB } & F: GTGTTGGATTCCGCCAGTGA & 366 & 61 & This study \\
\hline & R: TTCCGCCGCTACCTCTTCA & & & \\
\hline \multirow[t]{2}{*}{$\operatorname{mag} A$} & F: GGTGCTCTTTACATCATTGC & 1282 & 51 & $(17)$ \\
\hline & R: GCAATGGCCATTTGCGTTAG & & & \\
\hline \multirow[t]{2}{*}{$r m p A$} & F: GAGTATTGGTTGACAGCAGGAT & 250 & 53 & This study \\
\hline & R: AGCCGTGGATAATGGTTTACAA & & & \\
\hline \multirow[t]{2}{*}{ kfu } & F: ATAGTAGGCGAGCACCGAGA & 520 & 60 & $(18)$ \\
\hline & R: AGAACCTTCCTCGCTGAACA & & & \\
\hline \multirow[t]{2}{*}{ allS } & F: CCGAAACATTACGCACCTTT & 508 & 60 & $(18)$ \\
\hline & R: ATCACGAAGAGCCAGGTCAC & & & \\
\hline \multirow[t]{2}{*}{$y b t$} & F: GACGGAAACAGCACGGTAAA & 242 & 60 & $(17)$ \\
\hline & R: GAGCATAATAAGGCGAAAGA & & & \\
\hline \multirow[t]{2}{*}{$b / a_{\mathrm{CTX}-\mathrm{M} 15}$} & F: CGCTTTGCGATGTGCAG & 590 & 53 & This study \\
\hline & R: ACCGCGATATCGTTGGT & & & \\
\hline \multirow[t]{2}{*}{$b / a_{\mathrm{TEM}}$} & F: GAGTATTCAACATTTCCGTGTC & 800 & 54 & $(19)$ \\
\hline & R: TAATCAGTGAGGCACCTATCTC & & & \\
\hline \multirow[t]{2}{*}{$b l a_{\mathrm{SHV}}$} & F: AAGATCCACTATCGCCAGCAG & 200 & 60 & $(19)$ \\
\hline & R: ATTCAGTTCCGTTTCCCAGCGG & & & \\
\hline \multirow[t]{2}{*}{$b / a_{0 X A-48}$} & F-GCGTGGTTAAGGATGAACAC & 745 & 60 & $(19)$ \\
\hline & R-CATCAAGTTCAACCCAACCG & & & \\
\hline \multirow[t]{2}{*}{$b / a_{\mathrm{KPC}-1}$} & F: CGTCTAGTTCTGCTGTCTTG & 798 & 55 & $(20)$ \\
\hline & R:CTTGTCATCCTTGTTAGGCG & & & \\
\hline \multirow[t]{2}{*}{$b / a_{\mathrm{NDM}-1}$} & F: GGTTTGGCGATCTGGTTTTC & 621 & 54 & $(20)$ \\
\hline & R: CGGAATGGCTCATCACGATC & & & \\
\hline \multirow[t]{2}{*}{ Ompk36 group A } & F: GAAGGCGCTCTGTCTCCTA & 97 & 60 & $(12)$ \\
\hline & R: TGCCATCATAGATGTCATAGG & & & \\
\hline
\end{tabular}




\begin{tabular}{|lllll|}
\hline Primer name & Primer sequence $\left(\mathbf{5}^{\prime} \rightarrow \mathbf{3}^{\prime}\right)$ & $\begin{array}{l}\text { Amplicon } \\
\text { size }(\mathrm{bp})\end{array}$ & $\begin{array}{l}\text { Annealing } \\
\text { temperature }\left({ }^{\circ} \mathrm{C}\right)\end{array}$ & REF. \\
\hline Ompk36 group B & $\begin{array}{l}\text { F: CGGTCGTGGCGCGCAGAAA } \\
\text { R: GGTTGTTCTGA TCGTCGGTA }\end{array}$ & 125 & 64 & $(12)$ \\
\hline Ompk36 group C & F: CAACAACGGTCGT GGTTGGA & 144 & 62 & $(12)$ \\
& R: CCCAGTGCCGGAACACTATT & & & $(12)$ \\
\hline Ompk36 group D & F: GAAGGTACTTCTCCGACCAA & 283 & 62 & \\
& R: AATCAGATTCTCCGTTGCCG & & & \\
\hline
\end{tabular}

\section{Results}

\subsection{Phenotypic tests}

In this study, out of 477 K. pneumoniae isolates, 163 (34.2\%) were able to grow on tellurite-containing selective medium and were considered tellurite-resistant strains, so they were selected for the molecular identification test. In addition, 62 out of the 477 K. pneumoniae isolates (13\%) were reported with positive string test and hypermucoviscous phenotype.

\subsection{Molecular identification of hvKp}

On molecular identification (16), we found the iucA or iutA or peg344 as hvKp molecular markers in $21.4 \%(102 / 477)$ of total $K$. pneumoniae and $62.6 \%(102 / 163)$ of tellurite-resistant isolates. Thus, 45 isolates had only the iucA, 6 isolates had only the iutA, and 48 strains had both the iucA and iutA genes, all three genes (iucA, iutA and peg344) were detected simultaneously in only three hvKp isolates. Also 48\% (49/102) hvKp isolates were string positive. (Table 2) 
Table 2

Epidemiology, phenotypic and molecular characteristics of the hvKp isolates included in this study.

\begin{tabular}{|c|c|c|c|c|c|c|c|c|c|}
\hline Hospital & Ward & Source & $\begin{array}{l}\text { String } \\
\text { test }\end{array}$ & $\begin{array}{l}\text { Virulence } \\
\text { factor } \\
\text { genes }\end{array}$ & $\begin{array}{l}\text { Capsule } \\
\text { serotype }\end{array}$ & $\begin{array}{l}\text { ompK36 } \\
\text { typing }\end{array}$ & $\begin{array}{l}\text { Antibiotic } \\
\text { resistance } \\
\text { pattern }\end{array}$ & $\begin{array}{l}\text { Antibiotic } \\
\text { resistance } \\
\text { genes }\end{array}$ & $\begin{array}{l}\text { MIC } \\
(\mu \mathrm{g} / \mathrm{ml}) \\
\text { CAZ } \\
\text { IMI }\end{array}$ \\
\hline A & Internal & Tracheal & Neg & $\begin{array}{l}\text { iucA, } \\
\text { iutA, ybt }\end{array}$ & K20 & C & $\begin{array}{l}\text { CTX, GN, } \\
\text { IMI, FEP, } \\
\text { CRO, CAZ, } \\
\text { CIP, MRP, } \\
\text { AZM }\end{array}$ & $\begin{array}{l}\text { bla } \\
\text { blaM } \\
\text { bla }_{\mathrm{SHV}} \\
\text { ala } \\
\text { and } \\
\text { bla-M15 } \\
\text { OlXA-48 }\end{array}$ & ND ND \\
\hline A & ICU & Blood & Neg & $\begin{array}{l}\text { iucA, } \\
\text { iutA, ybt }\end{array}$ & K20 & C & $\begin{array}{l}\text { CTX, GN, } \\
\text { IMI, FEP, } \\
\text { CRO, CAZ, } \\
\text { CIP, MRP, } \\
\text { AZM }\end{array}$ & $\begin{array}{l}b / a_{\mathrm{TEM}}, \\
b a_{\mathrm{SHV}} \\
b / a_{\mathrm{CTX}-\mathrm{M} 15} \\
\text { and } \\
\text { bla } \\
\text { OXX-48 }\end{array}$ & ND ND \\
\hline A & Emergency & Urine & Pos & $\begin{array}{l}i u c A, k f u \\
y b t\end{array}$ & ND & B & ND & $b / a_{S H V}$ & ND ND \\
\hline A & ICU & Tracheal & Neg & iucA & K20 & A & $\begin{array}{l}\text { CTX, GN, } \\
\text { IMI, FEP, } \\
\text { CRO, AK, } \\
\text { CAZ, CIP, } \\
\text { MRP, AZM }\end{array}$ & $\begin{array}{l}\text { bla } a_{\mathrm{TEM}}, \\
b / a_{\mathrm{SHV}} \\
b / a_{\mathrm{OXA}-48} \\
\text { and } \\
\text { bla }_{\mathrm{NDM}-1}\end{array}$ & ND ND \\
\hline A & Surgery & Abscess & Pos & $i u c A, y b t$ & ND & A & $\begin{array}{l}\text { CTX, GN, } \\
\text { IMI, FEP, } \\
\text { CRO, AK, } \\
\text { CAZ, CIP, } \\
\text { MRP, AZM }\end{array}$ & $\begin{array}{l}b / a_{\mathrm{TEM}}, \\
b / a_{\mathrm{SHV}}, \\
b / a_{\mathrm{CTX}-\mathrm{M} 15}, \\
b / a_{\mathrm{OXA}-48} \\
\text { and } \\
\text { bla } \\
\text { NDMM-1 }_{\mathrm{N}}\end{array}$ & ND ND \\
\hline A & Surgery & Abscess & Pos & iucA & ND & C & ND & $b / a_{\mathrm{SHV}}$ & ND ND \\
\hline A & Surgery & $\begin{array}{l}\text { Abdominal } \\
\text { Secretions }\end{array}$ & Pos & $\begin{array}{l}\text { peg-344, } \\
\text { iucA, } \\
\text { iutA, iro, } \\
\text { ybt }\end{array}$ & K20 & C & $\begin{array}{l}\text { CTX, GN, } \\
\text { IMI, FEP, } \\
\text { CRO, CAZ, } \\
\text { CIP, MRP }\end{array}$ & $\begin{array}{l}b / a_{\mathrm{SHV}} \text { and } \\
b / a_{\mathrm{CTX}-\mathrm{M} 15}\end{array}$ & $16 \bowtie 2$ \\
\hline A & ICU & Tracheal & Neg & iucA & ND & A & $\begin{array}{l}\text { CTX, GN, } \\
\text { IMI, FEP,' } \\
\text { CRO, AK, } \\
\text { CAZ, CIP, } \\
\text { MRP, AZM }\end{array}$ & $\begin{array}{l}\text { bla } a_{\mathrm{TEM}}, \\
b / a_{\mathrm{SHV}} \\
b / a_{\mathrm{OXA}-48} \\
\text { and } \\
\text { bla }_{\mathrm{NDM}-1}\end{array}$ & ND ND \\
\hline A & ICU & Urine & Pos & $i u c A, k f u$ & ND & B & Susceptible & ND & ND ND \\
\hline A & ICU & Blood & $\mathrm{Neg}$ & $i u c A, y b t$ & ND & A & CTX & $b / a_{\mathrm{SHV}}$ & ND ND \\
\hline A & Orthopedics & $\begin{array}{l}\text { Synovial } \\
\text { fluid }\end{array}$ & Neg & $i u c A, k f u$ & ND & B & Susceptible & ND & ND ND \\
\hline
\end{tabular}

* M, male; F, female; ICU, Intensive Care Unit; Bal, bronchoalveolar lavage; CSF, cerebrospinal fluid; Pos, positive; Neg, negative; iuc and iut $A$, aerobactin genes; $y b t$, yersiniabactin; $r m p A$, regulator of mucoid phenotype; $K f u$, Klebsiella iron uptake; magA, mucoviscosity-associated gene; iroB, salmochelin iron uptake systems; allS, allantoin metabolism gene; peg-344, metabolic transporter; AK, amikacin; GN, gentamicin; CTX, cefotaxime; CAZ, ceftazidime; CRO, ceftriaxone; IMI, imipenem; MRP, meropenem; FEP, cefepime; CIP, ciprofloxacin;; AZM, aztreonam; MIC, minimum inhibitory concentration; ND, not determined. 


\begin{tabular}{|c|c|c|c|c|c|c|c|c|c|}
\hline Hospital & Ward & Source & $\begin{array}{l}\text { String } \\
\text { test }\end{array}$ & $\begin{array}{l}\text { Virulence } \\
\text { factor } \\
\text { genes }\end{array}$ & $\begin{array}{l}\text { Capsule } \\
\text { serotype }\end{array}$ & $\begin{array}{l}\text { ompK36 } \\
\text { typing }\end{array}$ & $\begin{array}{l}\text { Antibiotic } \\
\text { resistance } \\
\text { pattern }\end{array}$ & $\begin{array}{l}\text { Antibiotic } \\
\text { resistance } \\
\text { genes }\end{array}$ & $\begin{array}{l}\mathrm{MIC} \\
(\mu \mathrm{g} / \mathrm{ml}) \\
\mathrm{CAZ} \\
\mathrm{IMI}\end{array}$ \\
\hline$A$ & Out-patient & Urine & Pos & $i u c A, y b t$ & ND & C & $\mathrm{GN}, \mathrm{CAZ}$ & $\begin{array}{l}\text { bla } a_{\mathrm{TEM}} \text { and } \\
\text { bla } a_{\mathrm{SHV}}\end{array}$ & ND ND \\
\hline$A$ & ICU & Abscess & Neg & $\begin{array}{l}\text { iucA, } \\
\text { iutA }\end{array}$ & K20 & C & $\begin{array}{l}\text { CTX, GN, } \\
\text { IMI, CRO, } \\
\text { CAZ }\end{array}$ & $b / a_{\mathrm{TEM}}$ & ND ND \\
\hline$A$ & Internal & Urine & Neg & iucA & ND & A & ND & $\begin{array}{l}b / a_{\mathrm{TEM}} \text { and } \\
b / a_{\mathrm{SHV}}\end{array}$ & ND ND \\
\hline$A$ & ICU & Urine & Pos & $\begin{array}{l}\text { iucA, kfu, } \\
y b t\end{array}$ & K2 & C & $\begin{array}{l}\text { CTX, GN, } \\
\text { IMI, FEP, } \\
\text { CRO, AK, } \\
\text { CAZ, CIP, } \\
\text { MRP, AZM }\end{array}$ & $\begin{array}{l}\text { bla } a_{\mathrm{TEM}}, \\
\text { bla } \\
\text { bla } \\
\text { bla } a_{\mathrm{CTX}-\mathrm{M} 15} \\
\text { and } \\
\text { bla } \\
\text { OXX-48 }\end{array}$ & ND ND \\
\hline$A$ & Internal & Urine & Neg & $i u c A, y b t$ & ND & D & ND & $\begin{array}{l}\text { bla } a_{\mathrm{SHV}}, \\
\text { bla } \\
\text { and } \\
\text { bla }_{\mathrm{CXX}-\mathrm{M} 15} \\
\end{array}$ & ND ND \\
\hline$A$ & Out-patient & Urine & Pos & $i u c A, y b t$ & ND & C & Susceptible & ND & ND ND \\
\hline$A$ & Internal & Urine & Pos & $\begin{array}{l}\text { iucA, kfu, } \\
y b t\end{array}$ & ND & $\mathrm{C}$ & ND & 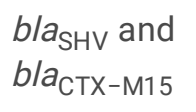 & ND ND \\
\hline$A$ & Out-patient & Urine & Neg & $\begin{array}{l}\text { iucA, } \\
\text { iutA, ybt, } \\
\text { rmpA }\end{array}$ & ND & C & $\begin{array}{l}\text { CTX, GN, } \\
\text { IMI, FEP, } \\
\text { CRO, AK, } \\
\text { CAZ, CIP, } \\
\text { MRP, AZM }\end{array}$ & $\begin{array}{l}\text { bla } a_{\mathrm{TEM}}, \\
\text { bla } \\
\text { and } \\
\text { ald } \\
\text { bla }_{\mathrm{NDM}-1}\end{array}$ & ND ND \\
\hline$A$ & Out-patient & Urine & Pos & $i u c A, k f u$ & ND & A & ND & $\begin{array}{l}\text { bla } a_{\mathrm{TEM}}, \\
b / a_{\mathrm{SHV}} \text { and } \\
b / a_{\mathrm{CTX}-\mathrm{M} 15}\end{array}$ & ND ND \\
\hline$A$ & Out-patient & Urine & Pos & $i u c A, y b t$ & K20 & B & ND & $\begin{array}{l}b / a_{\mathrm{SHV}} \text { and } \\
\text { bla }_{\mathrm{CTX}-\mathrm{M} 15}\end{array}$ & ND ND \\
\hline A & Internal & Urine & Neg & $i u c A, k f u$ & ND & C & ND & $b / a_{S H V}$ & ND ND \\
\hline$A$ & Internal & Sputum & Pos & $\begin{array}{l}\text { peg-344, } \\
\text { iucA, } \\
\text { iutA, ybt, } \\
\text { rmpA }\end{array}$ & $\mathrm{K} 20$ & C & CAZ & ND & ND ND \\
\hline$A$ & Surgery & $\begin{array}{l}\text { Abdominal } \\
\text { Secretions }\end{array}$ & Pos & iucA & ND & A & ND & $\begin{array}{l}b / a_{\mathrm{SHV}} \\
\text { bla }_{\mathrm{OXA}-48} \\
\text { and } \\
\text { bla }_{\mathrm{NDM}-1}\end{array}$ & ND ND \\
\hline
\end{tabular}

* M, male; F, female; ICU, Intensive Care Unit; Bal, bronchoalveolar lavage; CSF, cerebrospinal fluid; Pos, positive; Neg, negative; iuc and iutA, aerobactin genes; $y b t$, yersiniabactin; $r m p A$, regulator of mucoid phenotype; Kfu, Klebsiella iron uptake; magA, mucoviscosity-associated gene; iroB, salmochelin iron uptake systems; allS, allantoin metabolism gene; peg-344, metabolic transporter; AK, amikacin; GN, gentamicin; CTX, cefotaxime; CAZ, ceftazidime; CRO, ceftriaxone; IMI, imipenem; MRP, meropenem; FEP, cefepime; CIP, ciprofloxacin;; AZM, aztreonam; MIC, minimum inhibitory concentration; ND, not determined. 


\begin{tabular}{|c|c|c|c|c|c|c|c|c|c|}
\hline Hospital & Ward & Source & $\begin{array}{l}\text { String } \\
\text { test }\end{array}$ & $\begin{array}{l}\text { Virulence } \\
\text { factor } \\
\text { genes }\end{array}$ & $\begin{array}{l}\text { Capsule } \\
\text { serotype }\end{array}$ & $\begin{array}{l}\text { ompK36 } \\
\text { typing }\end{array}$ & $\begin{array}{l}\text { Antibiotic } \\
\text { resistance } \\
\text { pattern }\end{array}$ & $\begin{array}{l}\text { Antibiotic } \\
\text { resistance } \\
\text { genes }\end{array}$ & $\begin{array}{l}\mathrm{MIC} \\
(\mu \mathrm{g} / \mathrm{ml}) \\
\text { CAZ } \\
\text { IMI }\end{array}$ \\
\hline$A$ & Emergency & Urine & Pos & $\begin{array}{l}i u c A, k f u \\
y b t\end{array}$ & K2 & $\mathrm{C}$ & $\begin{array}{l}\text { CTX, GN, } \\
\text { IMI, FEP, } \\
\text { CRO, CAZ, } \\
\text { CIP, MRP, } \\
\text { AZM }\end{array}$ & $\begin{array}{l}\text { bla } \\
\text { bla } a_{\mathrm{SHV}}, \\
\text { bla }_{\mathrm{CTX}-\mathrm{M} 15} \\
\text { and } \\
\text { bla } \\
\text { OXA-48 }\end{array}$ & ND ND \\
\hline$A$ & Out-patient & Urine & Pos & $\begin{array}{l}i u c A, k f u \\
y b t\end{array}$ & $\mathrm{~K} 2$ & C & $\begin{array}{l}\text { CTX, GN, } \\
\text { IMI, FEP, } \\
\text { CRO, CAZ, } \\
\text { CIP, MRP, } \\
\text { AZM }\end{array}$ & $\begin{array}{l}\text { bla } \\
\text { bla } a_{\mathrm{SH}}, \\
\text { bla } \\
\text { and } \\
\text { bla }_{\mathrm{CTX}-\mathrm{M} 15-48}\end{array}$ & ND ND \\
\hline$A$ & Surgery & Urine & Pos & $\begin{array}{l}i u c A, k f u \\
y b t\end{array}$ & ND & $A$ & $\begin{array}{l}\text { CTX, GN, } \\
\text { IMI, FEP, } \\
\text { CRO, AK, } \\
\text { CAZ, CIP, } \\
\text { MRP, AZM }\end{array}$ & $\begin{array}{l}\text { bla } a_{\mathrm{TEM}}, \\
\text { bla } \\
\text { bla } \\
\text { bla } \\
\text { bla } a_{\mathrm{OXA}-\mathrm{M} 15}, \\
\text { and } \\
\text { bla } \\
\text { I }_{\mathrm{NDM}-1}\end{array}$ & ND ND \\
\hline A & Emergency & Urine & Pos & iucA, kfu & ND & A & ND & $b / a_{\mathrm{SHV}}$ & ND ND \\
\hline$A$ & ICU & Blood & Pos & $i u c A, k f u$ & ND & $D$ & $\begin{array}{l}\text { CTX, GN, } \\
\text { IMI, FEP, } \\
\text { CRO, AK, } \\
\text { CAZ, CIP, } \\
\text { MRP, AZM }\end{array}$ & $\begin{array}{l}\text { bla } a_{\mathrm{SHV}} \\
\text { bla } \\
\text { and } \\
\text { ala } 48 \\
\text { bla } a_{\mathrm{NDM}-1}\end{array}$ & ND ND \\
\hline$A$ & Orthopedics & Wound & $\mathrm{Neg}$ & $i u c A, y b t$ & ND & D & Susceptible & ND & ND ND \\
\hline$A$ & Graft & Urine & $\mathrm{Neg}$ & $\begin{array}{l}\text { iucA, kfu, } \\
\text { rmpA }\end{array}$ & K20 & C & $\begin{array}{l}\text { CTX, GN, } \\
\text { IMI, FEP, } \\
\text { CRO, CAZ, } \\
\text { CIP, MRP }\end{array}$ & $\begin{array}{l}\text { bla } a_{\mathrm{TEM}} \\
\text { bla } \\
\text { and } \\
\text { bla-M15 } \\
\text { blaXA-48 }\end{array}$ & ND ND \\
\hline$A$ & Out-patient & Urine & Pos & $i u c A, k f u$ & ND & C & $\begin{array}{l}\text { CTX, GN, } \\
\text { FEP, CRO, } \\
\text { CAZ, CIP }\end{array}$ & $\begin{array}{l}b / a_{\mathrm{TEM}}, \\
b / a_{\mathrm{SHV}} \text { and } \\
b / a_{\mathrm{CTX}-\mathrm{M} 15}\end{array}$ & ND ND \\
\hline$A$ & Graft & Urine & $\mathrm{Neg}$ & $\begin{array}{l}i u c A, y b t, \\
\text { alls, } \\
\text { rmpA }\end{array}$ & K20 & C & $\begin{array}{l}\text { CTX, GN, } \\
\text { IMI, FEP, } \\
\text { CRO, CAZ, } \\
\text { CIP, MRP, } \\
\text { AZM }\end{array}$ & $\begin{array}{l}\text { bla } a_{\mathrm{TEM}}, \\
\text { bla } a_{\mathrm{SHV}} \\
\text { bla }_{\mathrm{CTX}-\mathrm{M} 15}, \\
\text { bla }_{\mathrm{OXA}-48} \\
\text { bla }_{\mathrm{NDM}-1}\end{array}$ & $\triangle 164$ \\
\hline$A$ & ICU & Abscess & $\mathrm{Neg}$ & iucA & ND & C & Susceptible & ND & ND ND \\
\hline$A$ & ICU & Urine & $\mathrm{Neg}$ & iucA & ND & C & $\begin{array}{l}\text { CTX, FEP, } \\
\text { CAZ }\end{array}$ & $b / a_{\mathrm{SHV}}$ & ND ND \\
\hline
\end{tabular}

* M, male; F, female; ICU, Intensive Care Unit; Bal, bronchoalveolar lavage; CSF, cerebrospinal fluid; Pos, positive; Neg, negative; iuc and iutA, aerobactin genes; $y b t$, yersiniabactin; $r m p A$, regulator of mucoid phenotype; $K f u$, Klebsiella iron uptake; magA, mucoviscosity-associated gene; iroB, salmochelin iron uptake systems; alls, allantoin metabolism gene; peg-344, metabolic transporter; AK, amikacin; GN, gentamicin; CTX, cefotaxime; CAZ, ceftazidime; CRO, ceftriaxone; IMI, imipenem; MRP, meropenem; FEP, cefepime; CIP, ciprofloxacin;; AZM, aztreonam; MIC, minimum inhibitory concentration; ND, not determined. 


\begin{tabular}{|c|c|c|c|c|c|c|c|c|c|}
\hline Hospital & Ward & Source & $\begin{array}{l}\text { String } \\
\text { test }\end{array}$ & $\begin{array}{l}\text { Virulence } \\
\text { factor } \\
\text { genes }\end{array}$ & $\begin{array}{l}\text { Capsule } \\
\text { serotype }\end{array}$ & $\begin{array}{l}\text { ompK36 } \\
\text { typing }\end{array}$ & $\begin{array}{l}\text { Antibiotic } \\
\text { resistance } \\
\text { pattern }\end{array}$ & $\begin{array}{l}\text { Antibiotic } \\
\text { resistance } \\
\text { genes }\end{array}$ & $\begin{array}{l}\mathrm{MIC} \\
(\mu \mathrm{g} / \mathrm{ml}) \\
\text { CAZ } \\
\text { IMI }\end{array}$ \\
\hline A & ICU & Blood & $\mathrm{Neg}$ & iucA & ND & $\mathrm{D}$ & ND & $\begin{array}{l}\text { bla } a_{\mathrm{CTX}-\mathrm{M} 15} \\
\text { and } \\
\text { bla } \\
a_{\text {OXA-48 }}\end{array}$ & ND ND \\
\hline A & ICU & Abscess & Pos & $\begin{array}{l}\text { iucA, } \\
\text { iutA, } y b t \text {, } \\
\text { rmpA }\end{array}$ & K20 & C & ND & $\begin{array}{l}\text { bla } a_{\mathrm{TEM}} \\
\text { bla } \\
\text { and } \\
\text { and } \\
\text { bla }_{\mathrm{NDM}-1}\end{array}$ & $64 \rrbracket 4$ \\
\hline A & Internal & Tracheal & Neg & $i u c A, y b t$ & ND & A & $\begin{array}{l}\text { CTX, GN, } \\
\text { IMI, FEP, } \\
\text { CRO, AK, } \\
\text { CAZ, CIP, } \\
\text { MRP, AZM }\end{array}$ & $\begin{array}{l}\text { bla } a_{\mathrm{SHV}} \\
\text { bla } \\
\text { and } \\
\text { bla }_{\mathrm{C} \text { OXA-48 }}\end{array}$ & ND ND \\
\hline A & $\mathrm{ICU}$ & Tracheal & $\mathrm{Neg}$ & $\begin{array}{l}\text { iucA, } \\
\text { iutA }\end{array}$ & ND & C & $\begin{array}{l}\text { CTX, GN, } \\
\text { FEP, CRO, } \\
\text { AK, CAZ, } \\
\text { CIP }\end{array}$ & $b l a_{S H V}$ & ND ND \\
\hline A & Surgery & Wound & $\mathrm{Neg}$ & $\begin{array}{l}i u c A, k f u \\
y b t\end{array}$ & ND & C & $\begin{array}{l}\text { CTX, CRO, } \\
\text { CIP }\end{array}$ & $b l a_{\mathrm{SHV}}$ & ND ND \\
\hline A & Internal & Abscess & $\mathrm{Neg}$ & $i u c A, y b t$ & ND & D & $\begin{array}{l}\text { CTX, GN, } \\
\text { IMI, FEP, } \\
\text { CRO, CAZ, } \\
\text { CIP, MRP, } \\
\text { AZM }\end{array}$ & $\begin{array}{l}\text { bla } a_{\mathrm{TEM}}, \\
\text { bla } a_{\mathrm{SHV}} \\
\text { bla }_{\mathrm{CTX}-\mathrm{M} 15} \\
\text { and } \\
\text { bla }_{\mathrm{OXA}-48}\end{array}$ & ND ND \\
\hline A & Surgery & Abscess & $\mathrm{Neg}$ & $i u c A, y b t$ & ND & C & $\begin{array}{l}\text { CTX, GN, } \\
\text { IMI, FEP, } \\
\text { CRO, AK, } \\
\text { CAZ, CIP, } \\
\text { MRP, AZM }\end{array}$ & $\begin{array}{l}\text { bla } a_{\mathrm{TEM}} \\
\text { bla } \\
\text { and } \\
\text { bla-M15 } \\
\text { blaXA-48 }\end{array}$ & ND ND \\
\hline A & Emergency & Urine & $\mathrm{Neg}$ & $\begin{array}{l}i u c A, k f u \\
y b t\end{array}$ & ND & C & Susceptible & ND & ND ND \\
\hline A & Internal & Sputum & Pos & $\begin{array}{l}\text { iucA, } \\
\text { iutA, ybt }\end{array}$ & K20 & C & $\begin{array}{l}\text { CTX, GN, } \\
\text { FEP, CRO, } \\
\text { CAZ }\end{array}$ & $\begin{array}{l}b / a_{\mathrm{TEM}}, \\
b / a_{\mathrm{SHV}} \text { and } \\
b / a_{\mathrm{CTX}-\mathrm{M} 15}\end{array}$ & ND ND \\
\hline A & Out-patient & Urine & $\mathrm{Neg}$ & $\begin{array}{l}\text { iucA, kfu, } \\
y b t\end{array}$ & ND & $A$ & $\begin{array}{l}\text { CTX, GN, } \\
\text { IMI, FEP, } \\
\text { CRO, AK, } \\
\text { CAZ, CIP, } \\
\text { MRP }\end{array}$ & $\begin{array}{l}b / a_{\mathrm{CTX}-\mathrm{M} 15} \\
\text { and } \\
\text { bla } a_{\text {OXA-48 }}\end{array}$ & ND ND \\
\hline A & $\mathrm{ICU}$ & Abscess & Pos & $i u c A, y b t$ & ND & $\mathrm{D}$ & $\begin{array}{l}\text { CTX, GN, } \\
\text { IMI, FEP, } \\
\text { CRO, AK, } \\
\text { CAZ, CIP, } \\
\text { MRP, AZM }\end{array}$ & $\begin{array}{l}\text { bla } a_{\mathrm{SHV}}, \\
\text { bla }_{\mathrm{CTX}-\mathrm{M} 15,}, \\
\text { bla } a_{\mathrm{OXA}-48} \\
\text { and } \\
\text { bla }_{\mathrm{NDM}-1}\end{array}$ & $\otimes 168$ \\
\hline
\end{tabular}

* M, male; F, female; ICU, Intensive Care Unit; Bal, bronchoalveolar lavage; CSF, cerebrospinal fluid; Pos, positive; Neg, negative; iuc and iutA, aerobactin genes; $y b t$, yersiniabactin; $r m p A$, regulator of mucoid phenotype; Kfu, Klebsiella iron uptake; $m a g A$, mucoviscosity-associated gene; iroB, salmochelin iron uptake systems; allS, allantoin metabolism gene; peg-344, metabolic transporter; AK, amikacin; GN, gentamicin; CTX, cefotaxime; CAZ, ceftazidime; CRO, ceftriaxone; IMI, imipenem; MRP, meropenem; FEP, cefepime; CIP, ciprofloxacin;; AZM, aztreonam; MIC, minimum inhibitory concentration; ND, not determined. 


\begin{tabular}{|c|c|c|c|c|c|c|c|c|c|}
\hline \multirow[t]{2}{*}{ Hospital } & \multirow[t]{2}{*}{ Ward } & \multirow[t]{2}{*}{ Source } & \multirow[t]{2}{*}{$\begin{array}{l}\text { String } \\
\text { test }\end{array}$} & \multirow{2}{*}{$\begin{array}{l}\text { Virulence } \\
\text { factor } \\
\text { genes }\end{array}$} & \multirow[t]{2}{*}{$\begin{array}{l}\text { Capsule } \\
\text { serotype }\end{array}$} & \multirow[t]{2}{*}{$\begin{array}{l}\text { ompK36 } \\
\text { typing }\end{array}$} & \multirow{2}{*}{$\begin{array}{l}\text { Antibiotic } \\
\text { resistance } \\
\text { pattern }\end{array}$} & \multirow{2}{*}{$\begin{array}{l}\text { Antibiotic } \\
\text { resistance } \\
\text { genes }\end{array}$} & \multirow{2}{*}{$\begin{array}{l}\text { MIC } \\
(\mu \mathrm{g} / \mathrm{ml}) \\
\text { CAZ } \\
\text { IMI }\end{array}$} \\
\hline & & & & & & & & & \\
\hline$A$ & Emergency & Blood & Neg & iucA, kfu & ND & B & AK & ND & ND ND \\
\hline A & ICU & Tracheal & $\mathrm{Neg}$ & $i u c A, y b t$ & ND & $A$ & $\begin{array}{l}\text { CTX, GN, } \\
\text { IMI, FEP, } \\
\text { CRO, AK, } \\
\text { CAZ, CIP, } \\
\text { MRP, AZM }\end{array}$ & $\begin{array}{l}b / a_{\mathrm{CTX}-\mathrm{M} 15} \\
\text { and } \\
\text { bla }_{\mathrm{OXA}-48}\end{array}$ & ND ND \\
\hline A & ICU & Blood & Pos & $i u c A, y b t$ & ND & D & $\begin{array}{l}\text { CTX, GN, } \\
\text { IMI, FEP, } \\
\text { CRO, AK, } \\
\text { CAZ, CIP, } \\
\text { MRP, AZM }\end{array}$ & $\begin{array}{l}\text { bla } \\
\text { bla } \\
\text { and } \\
\text { and } \\
\text { bla } a_{\text {OXA-48 }}\end{array}$ & ND ND \\
\hline A & Neurology & Urine & Pos & $\begin{array}{l}\text { iucA, } \\
\text { iutA, } y b t \\
\text { rmpA }\end{array}$ & K20 & $C$ & $\begin{array}{l}\text { CTX, GN, } \\
\text { IMI, FEP, } \\
\text { CRO, CAZ, } \\
\text { CIP, MRP, } \\
\text { AZM }\end{array}$ & $\begin{array}{l}b / a_{\mathrm{TEM}}, \\
b / a_{\mathrm{SHV}}, \\
b / a_{\mathrm{CTX}-\mathrm{M} 15} \\
\text { and } \\
\text { bla } \\
\text { OXX-48 }\end{array}$ & ND ND \\
\hline A & Emergency & Urine & Pos & $\begin{array}{l}\text { iucA, } \\
\text { iutA, ybt, } \\
\text { rmpA }\end{array}$ & K20 & C & $\begin{array}{l}\text { CTX, GN, } \\
\text { IMI, FEP, } \\
\text { CRO, CAZ, } \\
\text { CIP, MRP, } \\
\text { AZM }\end{array}$ & $\begin{array}{l}b / a_{\mathrm{TEM}}, \\
b / a_{\mathrm{SHV}}, \\
b / a_{\mathrm{CTX}-\mathrm{M} 15} \\
\text { and } \\
\text { bla } \\
\text { OXX-48 }\end{array}$ & ND ND \\
\hline A & Internal & Wound & Pos & $\begin{array}{l}\text { iucA, } \\
\text { iutA, ybt, } \\
\text { rmpA }\end{array}$ & K20 & C & $\begin{array}{l}\text { CTX, GN, } \\
\text { IMI, FEP, } \\
\text { CRO, AK, } \\
\text { CAZ, CIP, } \\
\text { MRP, AZM }\end{array}$ & $\begin{array}{l}\text { bla } a_{\mathrm{TEM}}, \\
\text { bla } \\
\text { bla }_{\mathrm{SHV}}, \\
\text { and } \\
\text { bla } \\
\text { bleXA-48 }\end{array}$ & 162 \\
\hline A & Emergency & Urine & Pos & $\begin{array}{l}\operatorname{iucA}, \\
\text { iutA, } y b t \\
\text { rmpA }\end{array}$ & ND & $C$ & $\begin{array}{l}\text { CTX, GN, } \\
\text { IMI, FEP, } \\
\text { CRO, CAZ, } \\
\text { CIP, MRP, } \\
\text { AZM }\end{array}$ & $\begin{array}{l}\text { bla } a_{\mathrm{TEM}}, \\
b / a_{\mathrm{SHV}} \\
b / a_{\mathrm{CTX}-\mathrm{M} 15} \\
\text { and } \\
\text { bla } \\
a_{\mathrm{OXA}-48}\end{array}$ & $32 \varangle 4$ \\
\hline A & ICU & Bal & Pos & $\begin{array}{l}\text { iucA, } \\
\text { iutA, ybt, } \\
\text { rmpA }\end{array}$ & $\mathrm{K} 20$ & C & $\begin{array}{l}\text { CTX, GN, } \\
\text { IMI, FEP, } \\
\text { CRO, CAZ, } \\
\text { CIP, MRP, } \\
\text { AZM }\end{array}$ & $\begin{array}{l}\text { bla } \\
\text { blaM } \\
\text { bla } \\
\text { bla } \\
\text { and } \\
\text { and } \\
\text { bla } a_{\mathrm{OXA}-48}\end{array}$ & ND ND \\
\hline A & Surgery & Urine & Pos & $\begin{array}{l}\text { iucA, } \\
\text { iutA, ybt, } \\
\text { rmpA }\end{array}$ & K20 & C & $\begin{array}{l}\text { CTX, GN, } \\
\text { FEP, CRO, } \\
\text { CAZ, CIP, } \\
\text { MRP }\end{array}$ & $\begin{array}{l}b / a_{\mathrm{TEM}}, \\
b / a_{\mathrm{SHV}} \text { and } \\
b / a_{\mathrm{CTX}-\mathrm{M} 15}\end{array}$ & ND ND \\
\hline
\end{tabular}

* M, male; F, female; ICU, Intensive Care Unit; Bal, bronchoalveolar lavage; CSF, cerebrospinal fluid; Pos, positive; Neg, negative; iuc and iutA, aerobactin genes; $y b t$, yersiniabactin; $r m p A$, regulator of mucoid phenotype; Kfu, Klebsiella iron uptake; magA, mucoviscosity-associated gene; iroB, salmochelin iron uptake systems; allS, allantoin metabolism gene; peg-344, metabolic transporter; AK, amikacin; GN, gentamicin; CTX, cefotaxime; CAZ, ceftazidime; CRO, ceftriaxone; IMI, imipenem; MRP, meropenem; FEP, cefepime; CIP, ciprofloxacin;; AZM, aztreonam; MIC, minimum inhibitory concentration; ND, not determined. 


\begin{tabular}{|c|c|c|c|c|c|c|c|c|c|}
\hline Hospital & Ward & Source & $\begin{array}{l}\text { String } \\
\text { test }\end{array}$ & $\begin{array}{l}\text { Virulence } \\
\text { factor } \\
\text { genes }\end{array}$ & $\begin{array}{l}\text { Capsule } \\
\text { serotype }\end{array}$ & $\begin{array}{l}\text { ompK36 } \\
\text { typing }\end{array}$ & $\begin{array}{l}\text { Antibiotic } \\
\text { resistance } \\
\text { pattern }\end{array}$ & $\begin{array}{l}\text { Antibiotic } \\
\text { resistance } \\
\text { genes }\end{array}$ & $\begin{array}{l}\text { MIC } \\
(\mu \mathrm{g} / \mathrm{ml}) \\
\text { CAZ } \\
\text { IMI }\end{array}$ \\
\hline$A$ & ICU & Blood & Pos & $\begin{array}{l}\text { iucA, } \\
\text { iutA, ybt, } \\
\text { rmpA }\end{array}$ & K20 & C & $\begin{array}{l}\text { CTX, GN, } \\
\text { IMI, FEP, } \\
\text { CRO, AK, } \\
\text { CAZ, CIP, } \\
\text { MRP, AZM }\end{array}$ & $\begin{array}{l}\text { bla } a_{\mathrm{TEM}}, \\
b / a_{\mathrm{SHV}} \text { and } \\
b / a_{\mathrm{CTX}-\mathrm{M} 15}\end{array}$ & $\otimes 16 \varangle 4$ \\
\hline$A$ & ND & ND & Pos & $\begin{array}{l}\text { iucA, } \\
\text { iutA, ybt, } \\
\text { rmpA }\end{array}$ & K20 & C & $\begin{array}{l}\text { CTX, GN, } \\
\text { FEP, CRO, } \\
\text { CAZ, CIP }\end{array}$ & $\begin{array}{l}\text { bla } a_{\mathrm{TEM}}, \\
b / a_{\mathrm{SHV}} \text { and } \\
b / a_{\mathrm{CTX}-\mathrm{M} 15}\end{array}$ & ND ND \\
\hline A & ND & ND & Pos & $\begin{array}{l}\text { iucA, } \\
\text { iutA, } \\
\text { rmpA }\end{array}$ & ND & C & $\begin{array}{l}\text { CTX, GN, } \\
\text { IMI, FEP, } \\
\text { CRO, CAZ, } \\
\text { CIP, MRP, } \\
\text { AZM }\end{array}$ & $\begin{array}{l}b / a_{\mathrm{TEM}}, \\
b / a_{\mathrm{SHV}} \\
b / a_{\mathrm{CTX}-\mathrm{M} 15} \\
\text { and } \\
\text { bla } \\
\text { OXX-48 }\end{array}$ & ND ND \\
\hline A & ND & ND & Pos & $\begin{array}{l}\text { iucA, } \\
\text { iutA, } y b t \\
\text { rmpA }\end{array}$ & K20 & D & $\begin{array}{l}\text { CTX, GN, } \\
\text { IMI, FEP, } \\
\text { CRO, CAZ, } \\
\text { CIP, MRP, } \\
\text { AZM }\end{array}$ & $\begin{array}{l}\text { bla } a_{\mathrm{TEM}}, \\
\text { bla } \\
\text { and } \\
\text { bla-M15 } \\
\text { OlXA-48 }\end{array}$ & $64 \llbracket 4$ \\
\hline A & Emergency & Urine & Pos & $\begin{array}{l}\text { iucA, } \\
\text { iutA, ybt }\end{array}$ & K20 & C & $\begin{array}{l}\text { CTX, GN, } \\
\text { IMI, FEP, } \\
\text { CRO, CAZ, } \\
\text { CIP, MRP }\end{array}$ & $\begin{array}{l}b / a_{\mathrm{TEM}} \text { and } \\
b / a_{\mathrm{CTX}-\mathrm{M} 15}\end{array}$ & ND ND \\
\hline A & ICU & $\mathrm{CSF}$ & Pos & $\begin{array}{l}\text { iucA, } \\
\text { iutA, ybt, } \\
\text { rmpA }\end{array}$ & K20 & C & $\begin{array}{l}\text { CTX, GN, } \\
\text { FEP, CRO, } \\
\text { CAZ, CIP }\end{array}$ & $\begin{array}{l}b / a_{\mathrm{TEM}} \\
b / a_{\mathrm{SHV}} \text { and } \\
b / a_{\mathrm{CTX}-\mathrm{M} 15}\end{array}$ & ND ND \\
\hline A & ICU & Tracheal & Pos & $i u c A, y b t$ & K20 & D & $\begin{array}{l}\text { CTX, GN, } \\
\text { IMI, FEP, } \\
\text { CRO, CAZ, } \\
\text { CIP, MRP, } \\
\text { AZM }\end{array}$ & $\begin{array}{l}\text { bla } a_{\mathrm{TEM}}, \\
\text { bla } \\
\text { bla } \\
\text { bla } \\
\text { and } \\
\text { bla } \\
\text { OlXXA-48 }\end{array}$ & ND ND \\
\hline A & ICU & Blood & Pos & $\begin{array}{l}\text { iucA, } \\
\text { iutA, ybt, } \\
\text { rmpA }\end{array}$ & K20 & C & $\begin{array}{l}\text { CTX, FEP, } \\
\text { CRO, CAZ, } \\
\text { CIP }\end{array}$ & $\begin{array}{l}b / a_{\mathrm{TEM}} \\
b / a_{\mathrm{SHV}} \text { and } \\
b / a_{\mathrm{CTX}-\mathrm{M} 15}\end{array}$ & ND ND \\
\hline B & Infectious & Sputum & $\mathrm{Neg}$ & lutA, ybt & ND & C & $\begin{array}{l}\text { CTX, GN, } \\
\text { FEP, CRO, } \\
\text { CAZ, CIP }\end{array}$ & $b / a_{\mathrm{CTX}-\mathrm{M} 15}$ & ND ND \\
\hline B & ND & ND & Pos & $i u c A, y b t$ & ND & D & $\begin{array}{l}\text { CTX, GN, } \\
\text { IMI, FEP, } \\
\text { CRO, CAZ, } \\
\text { CIP, MRP, } \\
\text { AZM }\end{array}$ & 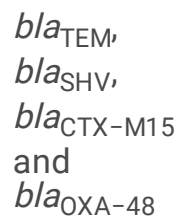 & 648 \\
\hline
\end{tabular}

* M, male; F, female; ICU, Intensive Care Unit; Bal, bronchoalveolar lavage; CSF, cerebrospinal fluid; Pos, positive; Neg, negative; iuc and iutA, aerobactin genes; $y b t$, yersiniabactin; $r m p A$, regulator of mucoid phenotype; Kfu, Klebsiella iron uptake; magA, mucoviscosity-associated gene; iroB, salmochelin iron uptake systems; allS, allantoin metabolism gene; peg-344, metabolic transporter; AK, amikacin; GN, gentamicin; CTX, cefotaxime; CAZ, ceftazidime; CRO, ceftriaxone; IMI, imipenem; MRP, meropenem; FEP, cefepime; CIP, ciprofloxacin;; AZM, aztreonam; MIC, minimum inhibitory concentration; ND, not determined. 


\begin{tabular}{|c|c|c|c|c|c|c|c|c|c|}
\hline Hospital & Ward & Source & $\begin{array}{l}\text { String } \\
\text { test }\end{array}$ & $\begin{array}{l}\text { Virulence } \\
\text { factor } \\
\text { genes }\end{array}$ & $\begin{array}{l}\text { Capsule } \\
\text { serotype }\end{array}$ & $\begin{array}{l}\text { ompK36 } \\
\text { typing }\end{array}$ & $\begin{array}{l}\text { Antibiotic } \\
\text { resistance } \\
\text { pattern }\end{array}$ & $\begin{array}{l}\text { Antibiotic } \\
\text { resistance } \\
\text { genes }\end{array}$ & $\begin{array}{l}\mathrm{MIC} \\
(\mu \mathrm{g} / \mathrm{ml}) \\
\mathrm{CAZ} \\
\mathrm{IMI}\end{array}$ \\
\hline B & ICU & Urine & $\mathrm{Neg}$ & $\begin{array}{l}\text { iucA, } \\
\text { iutA, } y b t \text {, } \\
\text { rmpA }\end{array}$ & K20 & C & $\begin{array}{l}\text { CTX, GN, } \\
\text { IMI, FEP, } \\
\text { CRO, CAZ, } \\
\text { CIP, MRP, } \\
\text { AZM }\end{array}$ & $\begin{array}{l}\text { bla } a_{\mathrm{TEM}}, \\
\text { bla } \\
\text { bla } \\
\text { bla } \\
\text { bla } a_{\mathrm{OXA}-\mathrm{M} 15}, \\
\text { and } \\
\text { bla } \\
\text { N }_{\mathrm{NDM}-1}\end{array}$ & 6416 \\
\hline B & Infectious & Urine & $\mathrm{Neg}$ & $\begin{array}{l}\text { iucA, } \\
\text { iutA, } y b t \text {, } \\
\text { rmpA }\end{array}$ & K20 & C & $\begin{array}{l}\text { CTX, GN, } \\
\text { IMI, FEP, } \\
\text { CRO, CAZ, } \\
\text { CIP, MRP, } \\
\text { AZM }\end{array}$ & $\begin{array}{l}\text { bla } a_{\mathrm{TEM}}, \\
\text { bla } a_{\mathrm{SHV}}, \\
\text { bla } \\
\text { bla } a_{\mathrm{OXA}-48}, \\
\text { and } \\
\text { bla } a_{\mathrm{NDM}-1}\end{array}$ & $\otimes 16 \varangle 4$ \\
\hline B & ICU & Tracheal & $\mathrm{Neg}$ & $\begin{array}{l}\text { iucA, } \\
\text { iutA, ybt, } \\
\text { rmpA }\end{array}$ & K20 & C & $\begin{array}{l}\text { CTX, GN, } \\
\text { IMI, FEP, } \\
\text { CRO, CAZ, } \\
\text { CIP, MRP, } \\
\text { AZM }\end{array}$ & $\begin{array}{l}\text { bla } a_{\mathrm{TEM}}, \\
\text { bla } \\
\text { bla } \\
\text { bla } \\
\text { bla } a_{\mathrm{OXA}-\mathrm{M} 15}, \\
\text { and } \\
\text { bla } \\
\text { N }_{\mathrm{NDM}-1}\end{array}$ & 644 \\
\hline B & ICU & Tracheal & Pos & $\begin{array}{l}\text { iucA, } \\
\text { iutA, } y b t \text {, } \\
\text { rmpA }\end{array}$ & K20 & C & $\begin{array}{l}\text { CTX, GN, } \\
\text { IMI, FEP, } \\
\text { CRO, AK, } \\
\text { CAZ, CIP, } \\
\text { MRP, AZM }\end{array}$ & $\begin{array}{l}\text { bla } a_{\mathrm{TEM}}, \\
\text { bla } a_{\mathrm{SHV}}, \\
\text { bla } \\
\text { and } \\
\text { bla-M15 } \\
\text { blaXA-48 }\end{array}$ & $64 \llbracket 4$ \\
\hline B & ICU & Urine & $\mathrm{Neg}$ & $\begin{array}{l}\text { iucA, } \\
\text { iutA, } y b t \\
\text { rmpA }\end{array}$ & K20 & C & $\begin{array}{l}\text { CTX, GN, } \\
\text { FEP, CRO, } \\
\text { CAZ, CIP, } \\
\text { AZM }\end{array}$ & $\begin{array}{l}\text { bla } a_{\mathrm{TEM}}, \\
b / a_{\mathrm{SHV}} \text { and } \\
b / a_{\mathrm{CTX}-\mathrm{M} 15}\end{array}$ & $\mathrm{ND} \otimes 2$ \\
\hline B & ND & ND & $\mathrm{Neg}$ & $\begin{array}{l}i u c A, k f u \\
y b t\end{array}$ & K20 & C & $\begin{array}{l}\text { CTX, GN, } \\
\text { IMI, FEP, } \\
\text { CRO, AK, } \\
\text { CAZ, CIP, } \\
\text { MRP, AZM }\end{array}$ & $\begin{array}{l}\text { bla } a_{\mathrm{TEM}}, \\
\text { bla } \\
\text { bla } \\
\text { bla } a_{\mathrm{CTX}-\mathrm{M} 15} \\
\text { and } \\
\text { bla } \\
\text { NDDM-1 }_{\mathrm{NDM}}\end{array}$ & $\otimes 16 \varangle 4$ \\
\hline B & ICU & Tracheal & $\mathrm{Neg}$ & $\begin{array}{l}\text { iucA, } \\
\text { iutA, ybt, } \\
\text { rmpA }\end{array}$ & K20 & C & $\begin{array}{l}\text { CTX, GN, } \\
\text { IMI, FEP, } \\
\text { CRO, AK, } \\
\text { CAZ, CIP, } \\
\text { MRP, AZM }\end{array}$ & $\begin{array}{l}\text { bla } a_{\mathrm{TEM}}, \\
\text { bla } \\
\text { bla } \\
\text { bla } \\
\text { bla } a_{\mathrm{OXA}-\mathrm{M} 15}, \\
\text { and } \\
\text { bla } \\
\text { N }_{\mathrm{NDM}-1}\end{array}$ & 644 \\
\hline B & ND & ND & Neg & $\begin{array}{l}\text { iucA, } \\
\text { iutA, ybt, } \\
\text { rmpA }\end{array}$ & K20 & C & $\begin{array}{l}\text { CTX, FEP, } \\
\text { CRO, AK, } \\
\text { CAZ, CIP }\end{array}$ & $\begin{array}{l}\text { bla } a_{\mathrm{TEM}}, \\
b / a_{\mathrm{SHV}} \text { and } \\
b / a_{\mathrm{CTX}-\mathrm{M} 15}\end{array}$ & ND ND \\
\hline
\end{tabular}

* M, male; F, female; ICU, Intensive Care Unit; Bal, bronchoalveolar lavage; CSF, cerebrospinal fluid; Pos, positive; Neg, negative; iuc and iutA, aerobactin genes; $y b t$, yersiniabactin; $r m p A$, regulator of mucoid phenotype; $K f u$, Klebsiella iron uptake; $m a g A$, mucoviscosity-associated gene; iroB, salmochelin iron uptake systems; allS, allantoin metabolism gene; peg-344, metabolic transporter; AK, amikacin; GN, gentamicin; CTX, cefotaxime; CAZ, ceftazidime; CRO, ceftriaxone; IMI, imipenem; MRP, meropenem; FEP, cefepime; CIP, ciprofloxacin;; AZM, aztreonam; MIC, minimum inhibitory concentration; ND, not determined. 


\begin{tabular}{|c|c|c|c|c|c|c|c|c|c|}
\hline Hospital & Ward & Source & $\begin{array}{l}\text { String } \\
\text { test }\end{array}$ & $\begin{array}{l}\text { Virulence } \\
\text { factor } \\
\text { genes }\end{array}$ & $\begin{array}{l}\text { Capsule } \\
\text { serotype }\end{array}$ & $\begin{array}{l}\text { ompK36 } \\
\text { typing }\end{array}$ & $\begin{array}{l}\text { Antibiotic } \\
\text { resistance } \\
\text { pattern }\end{array}$ & $\begin{array}{l}\text { Antibiotic } \\
\text { resistance } \\
\text { genes }\end{array}$ & $\begin{array}{l}\mathrm{MIC} \\
(\mu \mathrm{g} / \mathrm{ml}) \\
\mathrm{CAZ} \\
\text { IMI }\end{array}$ \\
\hline B & ICU & Blood & Pos & $\begin{array}{l}\text { peg-344, } \\
\text { luc, iutA, } \\
\text { iro, kfu, } \\
\text { ybt, } \\
\text { magA, } \\
\text { alls }\end{array}$ & $\mathrm{K} 1$ & C & $\begin{array}{l}\text { CTX, FEP, } \\
\text { CRO, CAZ, } \\
\text { CIP }\end{array}$ & $\begin{array}{l}b / a_{\mathrm{TEM}}, \\
b / a_{\mathrm{SHV}} \text { and } \\
b / a_{\mathrm{CTX}-\mathrm{M} 15}\end{array}$ & $\otimes 16 \bowtie 2$ \\
\hline B & ICU & Tracheal & $\mathrm{Neg}$ & $\begin{array}{l}\text { iucA, ybt, } \\
\text { rmpA }\end{array}$ & K20 & C & $\begin{array}{l}\text { CTX, FEP, } \\
\text { CRO, CAZ, } \\
\text { CIP }\end{array}$ & $\begin{array}{l}\text { bla } a_{\mathrm{TEM}}, \\
b / a_{\mathrm{SHV}}, \\
b / a_{\mathrm{CTX}-\mathrm{M} 15}\end{array}$ & ND 2 \\
\hline B & ICU & Tracheal & $\mathrm{Neg}$ & $\begin{array}{l}\text { iucA, } \\
\text { iutA, ybt, } \\
\text { rmpA }\end{array}$ & K20 & C & $\begin{array}{l}\text { CTX, FEP, } \\
\text { CRO, CAZ, } \\
\text { CIP }\end{array}$ & $\begin{array}{l}b / a_{\mathrm{TEM}}, \\
b / a_{\mathrm{SHV}} \text { and } \\
b / a_{\mathrm{CTX}-\mathrm{M} 15}\end{array}$ & ND 2 \\
\hline B & ICU & Urine & $\mathrm{Neg}$ & $\begin{array}{l}\text { iucA, } \\
\text { iutA, ybt, } \\
\text { rmpA }\end{array}$ & K20 & C & $\begin{array}{l}\text { CTX, FEP, } \\
\text { CRO, CAZ, } \\
\text { CIP }\end{array}$ & $\begin{array}{l}\text { bla } a_{\mathrm{TEM}}, \\
\text { bla } \\
\text { bla }_{\mathrm{SHV}}, \\
\mathrm{CTX}_{\mathrm{M} 15}\end{array}$ & ND 2 \\
\hline B & Surgery & Urine & Pos & $\begin{array}{l}\text { iucA, } \\
\text { iutA, } y b t \text {, } \\
\text { rmpA }\end{array}$ & $\mathrm{K} 20$ & C & $\begin{array}{l}\text { CTX, FEP, } \\
\text { CRO, CAZ, } \\
\text { CIP }\end{array}$ & $\begin{array}{l}\text { bla } a_{\mathrm{TEM}}, \\
b / a_{\mathrm{SHV}} \text { and } \\
b / a_{\mathrm{CTX}-\mathrm{M} 15}\end{array}$ & ND ND \\
\hline B & ICU & Tracheal & Pos & $\begin{array}{l}\text { iutA, iro, } \\
\text { rmpA }\end{array}$ & ND & C & $\begin{array}{l}\text { CTX, GN, } \\
\text { FEP, CRO, } \\
\text { AK, CAZ, } \\
\text { CIP }\end{array}$ & $b / a_{\mathrm{CTX}-\mathrm{M} 15}$ & ND ND \\
\hline B & ICU & Tracheal & $\mathrm{Neg}$ & iutA & ND & C & $\begin{array}{l}\text { CTX, GN, } \\
\text { IMI, FEP, } \\
\text { CRO, CAZ, } \\
\text { CIP, MRP }\end{array}$ & $\begin{array}{l}b / a_{\mathrm{TEM}} \text { and } \\
b / a_{\mathrm{SHV}}\end{array}$ & ND ND \\
\hline B & Internal & Urine & Pos & $\begin{array}{l}\text { iucA, } \\
\text { iutA, ybt, } \\
\text { rmpA }\end{array}$ & K20 & C & $\begin{array}{l}\text { CTX, GN, } \\
\text { IMI, FEP, } \\
\text { CRO, CAZ, } \\
\text { CIP, MRP }\end{array}$ & $\begin{array}{l}\text { bla } a_{\mathrm{TEM}}, \\
\text { bla } \\
\text { bla } \\
\text { bla } a_{\mathrm{CTX}-\mathrm{M} 15} \\
\text { and } \\
\text { bla } \\
\text { OXA-48 }\end{array}$ & ND 4 \\
\hline B & Internal & Tracheal & Pos & $\begin{array}{l}\text { iucA, } \\
\text { iutA, kfu, } \\
y b t \text {, } \\
\text { rmpA }\end{array}$ & $\mathrm{K} 20$ & D & $\begin{array}{l}\text { CTX, GN, } \\
\text { IMI, FEP, } \\
\text { CRO, CAZ, } \\
\text { CIP, MRP }\end{array}$ & $\begin{array}{l}\text { bla } a_{\mathrm{TEM}}, \\
\text { bla } \\
\text { bla } \\
\text { bla } a_{\mathrm{CTX}-\mathrm{M} 15} \\
\text { and } \\
\text { bla } \\
\text { OXA-48 }\end{array}$ & $\mathrm{ND} \otimes 4$ \\
\hline B & ND & ND & Pos & $\begin{array}{l}\text { iucA, } \\
\text { iutA, kfu, } \\
y b t, \\
\text { rmpA }\end{array}$ & K20 & C & $\begin{array}{l}\text { CTX, FEP, } \\
\text { CRO, AK, } \\
\text { CAZ, CIP }\end{array}$ & $\begin{array}{l}\text { bla } a_{\mathrm{SHV}} \text { and } \\
b / a_{\mathrm{CTX}-\mathrm{M} 15}\end{array}$ & ND ND \\
\hline
\end{tabular}

* M, male; F, female; ICU, Intensive Care Unit; Bal, bronchoalveolar lavage; CSF, cerebrospinal fluid; Pos, positive; Neg, negative; iuc and iutA, aerobactin genes; $y b t$, yersiniabactin; $r m p A$, regulator of mucoid phenotype; $K f u$, Klebsiella iron uptake; magA, mucoviscosity-associated gene; iroB, salmochelin iron uptake systems; alls, allantoin metabolism gene; peg-344, metabolic transporter; AK, amikacin; GN, gentamicin; CTX, cefotaxime; CAZ, ceftazidime; CRO, ceftriaxone; IMI, imipenem; MRP, meropenem; FEP, cefepime; CIP, ciprofloxacin;; AZM, aztreonam; MIC, minimum inhibitory concentration; ND, not determined. 


\begin{tabular}{|c|c|c|c|c|c|c|c|c|c|}
\hline Hospital & Ward & Source & $\begin{array}{l}\text { String } \\
\text { test }\end{array}$ & $\begin{array}{l}\text { Virulence } \\
\text { factor } \\
\text { genes }\end{array}$ & $\begin{array}{l}\text { Capsule } \\
\text { serotype }\end{array}$ & $\begin{array}{l}\text { ompK36 } \\
\text { typing }\end{array}$ & $\begin{array}{l}\text { Antibiotic } \\
\text { resistance } \\
\text { pattern }\end{array}$ & $\begin{array}{l}\text { Antibiotic } \\
\text { resistance } \\
\text { genes }\end{array}$ & $\begin{array}{l}\text { MIC } \\
(\mu \mathrm{g} / \mathrm{ml}) \\
\text { CAZ } \\
\text { IMI }\end{array}$ \\
\hline B & ND & ND & $\mathrm{Neg}$ & $\begin{array}{l}\text { iucA, } \\
\text { iutA, } y b t \text {, } \\
\text { rmpA }\end{array}$ & K20 & C & $\begin{array}{l}\text { CTX, GN, } \\
\text { FEP, CRO, } \\
\text { CAZ, CIP }\end{array}$ & $\begin{array}{l}b / a_{\mathrm{SHV}} \text { and } \\
b / a_{\mathrm{CTX}-\mathrm{M} 15}\end{array}$ & ND ND \\
\hline B & ICU & Tracheal & Pos & $\begin{array}{l}\text { iucA, } \\
\text { iutA, } y b t \text {, } \\
\text { rmpA }\end{array}$ & K20 & C & $\begin{array}{l}\text { CTX, GN, } \\
\text { IMI, FEP, } \\
\text { CRO, CAZ, } \\
\text { CIP, MRP, } \\
\text { AZM }\end{array}$ & $\begin{array}{l}\text { bla } \\
\text { bleM, } \\
\text { bla } \\
\text { bla } \\
\text { blaX-M15, } \\
\text { bla } a_{\mathrm{OXA}-48} \\
\text { and } \\
\text { bla }_{\mathrm{NDM}-1}\end{array}$ & $32 \varangle 4$ \\
\hline B & Infectious & Tracheal & Neg & iutA, ybt & ND & A & $\begin{array}{l}\text { CTX, GN, } \\
\text { IMI, FEP, } \\
\text { CRO, AK, } \\
\text { CAZ, CIP, } \\
\text { MRP, AZM }\end{array}$ & $\begin{array}{l}\text { bla } \\
\text { blaM, } \\
\text { bla } \\
\text { bla } a_{\mathrm{CTX}-\mathrm{M} 15}, \\
\text { bla } \\
\text { and } \\
\text { and } \\
\text { bla } \\
\text { NDM-1 }_{\mathrm{NDM}}\end{array}$ & $64 \otimes 4$ \\
\hline B & ICU & Tracheal & $\mathrm{Neg}$ & $\begin{array}{l}\text { iucA, ybt, } \\
\text { rmpA }\end{array}$ & K20 & C & $\begin{array}{l}\text { CTX, GN, } \\
\text { IMI, FEP, } \\
\text { CRO, AK, } \\
\text { CAZ, CIP, } \\
\text { MRP, AZM }\end{array}$ & $\begin{array}{l}\text { bla } \\
\text { bleM, } \\
\text { bla } \\
\text { bla } \\
\text { blX-M15, } \\
\text { bla } a_{\mathrm{OXA}-48} \\
\text { and } \\
\text { bla }_{\mathrm{NDM}-1}\end{array}$ & 324 \\
\hline B & ICU & Tracheal & $\mathrm{Neg}$ & iutA & K20 & A & $\begin{array}{l}\text { CTX, GN, } \\
\text { IMI, FEP, } \\
\text { CRO, AK, } \\
\text { CAZ, CIP, } \\
\text { MRP, AZM }\end{array}$ & $\begin{array}{l}\text { bla } a_{\mathrm{TEM}}, \\
\text { bla } \\
\text { bla } \\
\text { bla } \\
\text { and } \\
\text { bla } \\
\text { la }_{\mathrm{NDM}-1}\end{array}$ & 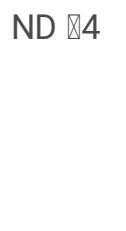 \\
\hline B & ICU & Tracheal & $\mathrm{Neg}$ & $\begin{array}{l}\text { iucA, ybt, } \\
\text { rmpA }\end{array}$ & K20 & C & $\begin{array}{l}\text { CTX, GN, } \\
\text { IMI, FEP, } \\
\text { CRO, AK, } \\
\text { CAZ, CIP, } \\
\text { MRP, AZM }\end{array}$ & $\begin{array}{l}\text { bla } \\
\text { bleM, } \\
\text { bla } \\
\text { bla }_{\mathrm{SHX}-\mathrm{M} 15}, \\
\text { bla } \\
\text { and } \\
\text { and } \\
\text { bla }_{\mathrm{NDM}-1}\end{array}$ & $64 \otimes 4$ \\
\hline B & ICU & Tracheal & Neg & $\begin{array}{l}\text { iucA, } \\
\text { iutA, ybt, } \\
\text { rmpA }\end{array}$ & K20 & C & $\begin{array}{l}\text { CTX, GN, } \\
\text { IMI, FEP, } \\
\text { CRO, AK, } \\
\text { CAZ, CIP, } \\
\text { MRP, AZM }\end{array}$ & $\begin{array}{l}b / a_{\mathrm{TEM}}, \\
\text { bla } \\
\text { bla } \\
\text { bla } \\
\text { bla-M15, } \\
\text { and } \\
\text { and } \\
\text { bla } a_{\mathrm{NDM}-1}\end{array}$ & ND $\otimes 1$ \\
\hline
\end{tabular}

* M, male; F, female; ICU, Intensive Care Unit; Bal, bronchoalveolar lavage; CSF, cerebrospinal fluid; Pos, positive; Neg, negative; iuc and iutA, aerobactin genes; $y b t$, yersiniabactin; $r m p A$, regulator of mucoid phenotype; Kfu, Klebsiella iron uptake; magA, mucoviscosity-associated gene; iroB, salmochelin iron uptake systems; allS, allantoin metabolism gene; peg-344, metabolic transporter; AK, amikacin; GN, gentamicin; CTX, cefotaxime; CAZ, ceftazidime; CRO, ceftriaxone; IMI, imipenem; MRP, meropenem; FEP, cefepime; CIP, ciprofloxacin;; AZM, aztreonam; MIC, minimum inhibitory concentration; ND, not determined. 


\begin{tabular}{|c|c|c|c|c|c|c|c|c|c|}
\hline Hospital & Ward & Source & $\begin{array}{l}\text { String } \\
\text { test }\end{array}$ & $\begin{array}{l}\text { Virulence } \\
\text { factor } \\
\text { genes }\end{array}$ & $\begin{array}{l}\text { Capsule } \\
\text { serotype }\end{array}$ & $\begin{array}{l}\text { ompK36 } \\
\text { typing }\end{array}$ & $\begin{array}{l}\text { Antibiotic } \\
\text { resistance } \\
\text { pattern }\end{array}$ & $\begin{array}{l}\text { Antibiotic } \\
\text { resistance } \\
\text { genes }\end{array}$ & $\begin{array}{l}\mathrm{MIC} \\
(\mu \mathrm{g} / \mathrm{ml}) \\
\text { CAZ } \\
\text { IMI }\end{array}$ \\
\hline B & ICU & Tracheal & Neg & $\begin{array}{l}\text { iucA, } \\
\text { iutA, ybt, } \\
\text { rmpA }\end{array}$ & K20 & C & $\begin{array}{l}\text { CTX, GN, } \\
\text { IMI, FEP, } \\
\text { CRO, CAZ, } \\
\text { CIP, MRP, } \\
\text { AZM }\end{array}$ & $\begin{array}{l}\text { bla } \\
\text { blaM, } \\
\text { bla } \\
\text { bla } \\
\text { bla } \\
\text { bla } a_{\mathrm{OXA}-48}, \\
\text { and } \\
\text { bla }_{\mathrm{NDM}-1}\end{array}$ & ND 16 \\
\hline B & ICU & CSF & $\mathrm{Neg}$ & $\begin{array}{l}\text { iucA, } \\
\text { iutA, ybt, } \\
\text { rmpA }\end{array}$ & K20 & C & $\begin{array}{l}\text { CTX, GN, } \\
\text { IMI, FEP, } \\
\text { CRO, CAZ, } \\
\text { CIP, MRP, } \\
\text { AZM }\end{array}$ & $\begin{array}{l}\text { bla } a_{\mathrm{TEM}}, \\
\text { bla } \\
\text { bla } \\
\text { bla } a_{\mathrm{CTX}-\mathrm{M} 15}, \\
\text { bla } \\
\text { and } \\
\text { bla } a_{\mathrm{NDM}-1}\end{array}$ & $128 \llbracket 4$ \\
\hline B & ICU & Urine & Neg & $\begin{array}{l}\text { iucA, } \\
\text { iutA, ybt, } \\
\text { rmpA }\end{array}$ & K20 & C & $\begin{array}{l}\text { CTX, GN, } \\
\text { IMI, FEP, } \\
\text { CRO, CAZ, } \\
\text { CIP, MRP, } \\
\text { AZM }\end{array}$ & $\begin{array}{l}\text { bla } \\
\text { blaM, } \\
\text { bla } \\
\text { bla } \\
\text { bla } \\
\text { bla } a_{\mathrm{OXA}-48}{ }^{2}, \\
\text { and } \\
\text { bla }_{\mathrm{NDM}-1}\end{array}$ & $64 \llbracket 4$ \\
\hline B & ICU & Bal & Pos & $\begin{array}{l}\text { iucA, } \\
\text { iutA, ybt, } \\
\text { rmpA }\end{array}$ & K20 & C & $\begin{array}{l}\text { CTX, GN, } \\
\text { IMI, FEP, } \\
\text { CRO, AK, } \\
\text { CAZ, CIP, } \\
\text { MRP, AZM }\end{array}$ & $\begin{array}{l}\text { bla } \\
\text { blaM, } \\
\text { bla } \\
\text { bla } \\
\text { bla } a_{\mathrm{OXA}-48} \\
\text { and } \\
\text { bla } \\
\text { NDM-1 }\end{array}$ & ND 4 \\
\hline B & ICU & Tracheal & $\mathrm{Neg}$ & $\begin{array}{l}\text { iucA, } \\
\text { iutA, ybt, } \\
\text { rmpA }\end{array}$ & K20 & C & $\begin{array}{l}\text { CTX, GN, } \\
\text { IMI, FEP, } \\
\text { CRO, CAZ, } \\
\text { CIP, MRP, } \\
\text { AZM }\end{array}$ & $\begin{array}{l}\text { bla } a_{\mathrm{TEM}}, \\
\text { bla } \\
\text { bla } \\
\text { bla } a_{\mathrm{CTX}-\mathrm{M} 15}, \\
\text { bla } \\
\text { and } \\
\text { bla } a_{\mathrm{NDM}-1}\end{array}$ & $64 \llbracket 4$ \\
\hline B & ICU & Tracheal & Pos & $\begin{array}{l}\text { iucA, } \\
\text { iutA, ybt, } \\
\text { rmpA }\end{array}$ & K20 & C & $\begin{array}{l}\text { CTX, GN, } \\
\text { IMI, FEP, } \\
\text { CRO, CAZ, } \\
\text { CIP, MRP, } \\
\text { AZM }\end{array}$ & $\begin{array}{l}\text { bla } \\
\text { blaM, } \\
\text { bla } \\
\text { bla } \\
\text { bla-M15, } \\
\text { blaXA-48 } \\
\text { and } \\
\text { bla }_{\mathrm{NDM}-1}\end{array}$ & $N D \otimes 4$ \\
\hline B & Surgery & Blood & Neg & $\begin{array}{l}\text { iucA, } \\
\text { iutA, ybt, } \\
\text { rmpA }\end{array}$ & K20 & C & $\begin{array}{l}\text { CTX, GN, } \\
\text { IMI, FEP, } \\
\text { CRO, AK, } \\
\text { CAZ, CIP, } \\
\text { MRP, AZM }\end{array}$ & $\begin{array}{l}\text { bla } \\
\text { bleM, } \\
\text { bla } \\
\text { bla } a_{\mathrm{CTX}-\mathrm{M} 15}, \\
\text { bla } \\
\text { and } \\
\text { and } \\
\text { bla } \\
\text { N }_{\mathrm{NDM}-1}\end{array}$ & ND 256 \\
\hline \multicolumn{10}{|c|}{$\begin{array}{l}\text { * M, male; F, female; ICU, Intensive Care Unit; Bal, bronchoalveolar lavage; CSF, cerebrospinal fluid; Pos, positive; Neg, } \\
\text { negative; iuc and iutA, aerobactin genes; ybt, yersiniabactin; } r m p A \text {, regulator of mucoid phenotype; Kfu, Klebsiella iron uptake; } \\
\text { magA, mucoviscosity-associated gene; iroB, salmochelin iron uptake systems; allS, allantoin metabolism gene; peg-344, } \\
\text { metabolic transporter; AK, amikacin; GN, gentamicin; CTX, cefotaxime; CAZ, ceftazidime; CRO, ceftriaxone; IMI, imipenem; } \\
\text { MRP, meropenem; FEP, cefepime; CIP, ciprofloxacin;; AZM, aztreonam; MIC, minimum inhibitory concentration; ND, not } \\
\text { determined. }\end{array}$} \\
\hline
\end{tabular}




\begin{tabular}{|c|c|c|c|c|c|c|c|c|c|}
\hline Hospital & Ward & Source & $\begin{array}{l}\text { String } \\
\text { test }\end{array}$ & $\begin{array}{l}\text { Virulence } \\
\text { factor } \\
\text { genes }\end{array}$ & $\begin{array}{l}\text { Capsule } \\
\text { serotype }\end{array}$ & $\begin{array}{l}\text { ompK36 } \\
\text { typing }\end{array}$ & $\begin{array}{l}\text { Antibiotic } \\
\text { resistance } \\
\text { pattern }\end{array}$ & $\begin{array}{l}\text { Antibiotic } \\
\text { resistance } \\
\text { genes }\end{array}$ & $\begin{array}{l}\text { MIC } \\
(\mu \mathrm{g} / \mathrm{ml}) \\
\text { CAZ } \\
\text { IMI }\end{array}$ \\
\hline B & ICU & Tracheal & $\mathrm{Neg}$ & $\begin{array}{l}\text { iucA, } \\
\text { iutA, ybt, } \\
\text { rmpA }\end{array}$ & K20 & C & $\begin{array}{l}\text { CTX, GN, } \\
\text { IMI, FEP, } \\
\text { CRO, CAZ, } \\
\text { CIP, MRP, } \\
\text { AZM }\end{array}$ & $\begin{array}{l}\text { bla } \\
\text { bleM, } \\
\text { bla } \\
\text { bla }_{\mathrm{SHX}-\mathrm{M} 15}, \\
\text { bla } \\
\text { and } \\
\text { and } \\
\text { bla }_{\mathrm{NDM}-1}\end{array}$ & ND 16 \\
\hline B & Surgery & Wound & $\mathrm{Neg}$ & $\begin{array}{l}\text { iucA, } \\
\text { iutA, ybt, } \\
\text { rmpA }\end{array}$ & $\mathrm{K} 20$ & C & $\begin{array}{l}\text { CTX, GN, } \\
\text { IMI, FEP, } \\
\text { CRO, CAZ, } \\
\text { CIP, MRP, } \\
\text { AZM }\end{array}$ & $\begin{array}{l}\text { bla } \\
\text { bla } \\
\text { bla } \\
\text { bla } \\
\text { bla } a_{\mathrm{OXA}-\mathrm{M} 15} \\
\text { and } \\
\text { bla } \\
\text { N/NM-1 }\end{array}$ & ND ND \\
\hline B & $\mathrm{ICU}$ & Tracheal & Pos & $\begin{array}{l}\text { iucA, } \\
\text { iutA, iro, } \\
\text { ybt, } \\
\text { rmpA }\end{array}$ & $\mathrm{K} 20$ & C & $\begin{array}{l}\text { CTX, GN, } \\
\text { IMI, FEP, } \\
\text { CRO, AK, } \\
\text { CAZ, CIP, } \\
\text { MRP, AZM }\end{array}$ & 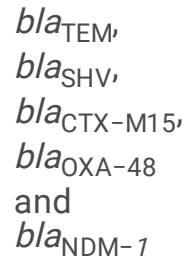 & ND 8 \\
\hline B & ICU & Tracheal & Neg & $\begin{array}{l}\text { iucA, } \\
\text { iutA, ybt, } \\
\text { rmpA }\end{array}$ & K20 & C & $\begin{array}{l}\text { CTX, GN, } \\
\text { IMI, FEP, } \\
\text { CRO, CAZ, } \\
\text { CIP, MRP, } \\
\text { AZM }\end{array}$ & $\begin{array}{l}\text { bla } \\
\text { bleM, } \\
\text { bla } \\
\text { bla } \\
\text { bla-M15, } \\
\text { bla } a_{\mathrm{OXA}-48} \\
\text { and } \\
\text { bla }_{\mathrm{NDM}-1}\end{array}$ & ND 8 \\
\hline B & $\mathrm{ICU}$ & Tracheal & $\mathrm{Neg}$ & $\begin{array}{l}\text { iucA, } \\
\text { iutA, ybt, } \\
\text { rmpA }\end{array}$ & $\mathrm{K} 20$ & C & $\begin{array}{l}\text { CTX, GN, } \\
\text { IMI, FEP, } \\
\text { CRO, CAZ, } \\
\text { CIP, MRP, } \\
\text { AZM }\end{array}$ & $\begin{array}{l}\text { bla } \\
\text { bla } \\
\text { bla } \\
\text { blaXA-48 } \\
\text { and } \\
\text { bla } \\
\text { NDM-1 }\end{array}$ & ND 8 \\
\hline \multicolumn{10}{|c|}{$\begin{array}{l}\text { * M, male; F, female; ICU, Intensive Care Unit; Bal, bronchoalveolar lavage; CSF, cerebrospinal fluid; Pos, positive; Neg, } \\
\text { negative; iuc and iutA, aerobactin genes; ybt, yersiniabactin; rmpA, regulator of mucoid phenotype; Kfu, Klebsiella iron uptake; } \\
\text { magA, mucoviscosity-associated gene; iroB, salmochelin iron uptake systems; allS, allantoin metabolism gene; peg-344, } \\
\text { metabolic transporter; AK, amikacin; GN, gentamicin; CTX, cefotaxime; CAZ, ceftazidime; CRO, ceftriaxone; IMI, imipenem; } \\
\text { MRP, meropenem; FEP, cefepime; CIP, ciprofloxacin;; AZM, aztreonam; MIC, minimum inhibitory concentration; ND, not } \\
\text { determined. }\end{array}$} \\
\hline
\end{tabular}

\subsection{Demographic data}

In this collection, 61.7\% (63/102) and 38.2\% (39/102) of hvKp strains were isolated from hospital A and B, respectively. Most of hvKp isolates were obtained from urine (33.3\%, 34/102), followed by tracheal $(27.5 \%, 28 / 102)$, blood $(9.8 \%, 10 / 102)$ and abscess $(7.8 \%, 8 / 102)$ specimens. Also among hospital wards, most hvKps were obtained from patients admitted to intensive care unit (ICU) (46.1\%), internal (11.8\%), surgical (10.8\%) and emergency (7.8\%) wards. Fifty-eight (56.9\%) were men and 36 (35.5\%) were women, most of whom were over 60 years of age (45.1\%). (Table 2).

\subsection{Antimicrobial susceptibility testing}

In our study, we investigated the antimicrobial susceptibility profile in 90 hvKp isolates. Table 2 shows the resistance profile of each hvKp isolate to the antibiotics used in this study. The highest rate of antibiotic resistance was related to ampicillin (100\%), followed by cefotaxime and ceftazidime (90\%). The lowest rate of resistance was found in amikacin (34.4\%). The resistance 
rates to other antibiotics including ceftriaxone, cefepime, ciprofloxacin, gentamicin, meropenem, imipenem and aztreonam were $87.7 \%, 86.6 \%, 85.5 \%, 78.8 \%, 66.6 \%, 66.6 \%$ and $58.8 \%$ respectively. In addition, $76.6 \%$ of the isolates $(69 / 90)$ were resistant to at least three classes of antibiotics and were defined as multidrug resistant (MDR) (21). Finally, the MIC of some resistant hvKp to the antibiotics ceftazidime and imipenem was determined by broth dilution method. All 23 isolates selected for ceftazidime had MIC $\geq 16$ : for 6 isolates $\mathrm{MIC} \otimes 16,2$ isolates $\mathrm{MIC}=16,3$ isolates $\mathrm{MIC}=32,11$ isolates $\mathrm{MIC}=64$, one isolate $\mathrm{MIC}=128$. Of the 39 resistant hvKp isolates, $79.5 \%$ had a MIC above the CLSI resistance criteria for imipenem (MIC $\geq 4$ ): for 16 isolates MIC $₫ 4,6$ isolates $\mathrm{MIC}=4,5$ isolates $\mathrm{MIC}=8,3$ isolates $\mathrm{MIC}=16$, one isolate $\mathrm{MIC}=256.4$ isolates $(10.2 \%)$ were classified as intermediate

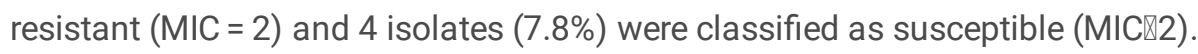

\subsection{Capsular genotyping and detection of virulence genes, and antimicrobial resistance genes}

Capsular genotyping (K genotyping) of hvKp isolates showed that capsular serotype K20 was detected in more than half of the hvKp strains (54.9\%). K2 and K1 were identified in only 3 (2.9\%) and one isolate (1\%), respectively, while K5, K54 and K57 were not detected in any of the hvKp isolates. In addition, 42 isolates (41.2\%) did not belong to serotypes $\mathrm{K} 1, \mathrm{~K} 2, \mathrm{~K} 5, \mathrm{~K} 20, \mathrm{~K} 54$ and K57 as shown in Table 2.

PCR for virulence-associated genes revealed that $y b t(77.5 \%)$ was the most common virulence factor gene after iucA. The other virulence factor genes including $r m p A$, iroB, magA, $k f u$ and allS were detected in $48 \%, 3.9 \%, 0.98,21.6 \%$ and $1.96 \%$ hvKp isolates, respectively. (Table 2)

The distribution of Extended spectrum beta-lactamases (ESBLs) and carbapenemase genes among hvKp isolates is shown in Table 2. The results showed that $92.2 \%$ (94/102) hvKp isolates carried at least one antibiotic resistance gene and only $7.8 \%$ $(8 / 102)$ had no resistance gene. The bla $a_{S H V}$ was the most common beta-lactamase gene (81.4\%), followed by b/a $a_{\mathrm{CTX}-\mathrm{M} 15}$ (75.5\%) and bla $a_{\mathrm{TEM}}(67.6 \%)$. Also, PCR amplification of carbapenemase genes showed that bla $a_{0 \mathrm{XA}-48}(33.7 \%)$ was the dominant genotype of carbapenem-resistant strains, followed by b/a $\mathrm{NDM}_{\mathrm{N}-1}(32.3 \%)$, while $b / a_{\mathrm{KPC}-1}$ was not detected in any hvKp isolate. Thus, 56.8\% (58/102) of the hvKp isolates carried ESBL and carbapenemase simultaneously and bla $a_{\mathrm{TEM}}, b / a_{\mathrm{SHV}}, b / a_{\mathrm{CTX}-\mathrm{M} 15}$,

$b / a_{\mathrm{OXA}-48}, b / a_{\mathrm{NDM}-1}$ was the predominant MDR-hvKp genotype $39.6 \%(23 / 58)$. On the other hand, as shown in Table 2, more than 2 virulence factor genes were detected simultaneously in the majority of resistant hvKp strains and virulence profiles including: iucA, iutA, ybt, rmpA genes have been reported in 40.4\% (38/94) resistant hvKp isolates.

\section{6. ompK36 typing}

PCR-based ompK36 typing revealed that ompK36 group C was the most common type with $70.6 \%(72 / 102)$ frequency. The prevalence of the other types, including ompK36 groups A, B, and C, was (14/102) 13.7\%, (5/102) 4.9\%, and 10.8\% (11/102), respectively (Table 2 ).

\subsection{Nucleotide accession numbers}

The accession numbers of bla $a_{\mathrm{OXA}-48}, b / a_{\mathrm{NDM}-1}$, iutA, iucA and peg344 are MZ245618, MZ245619, MZ245620, MZ245621 and MZ245622, respectively in GenBank database.

\section{Discussion}

Because of the importance of hvKp isolates in human infections, especially in people without underlying disease and immunodeficiency, it is necessary to use an appropriate laboratory method to obtain an accurate diagnosis of these strains. Previously, the string test was used as a phenotypic method to identify hvKp isolates $(22,23)$. According to our results, only 62 out of 477 K. pneumoniae and 49 out of 102 hvKp isolates showed string test positive. Thus, this result indicates that the string test is not a reliable rapid test for hvKp detection, and these results are in agreement with the findings of Russo et al. and Parrott et al. $(16,24)$. On the other hand, we used tellurite resistance in clinical strains for the first time for phenotypic isolation of hvKps. Of 162 tellurite-resistant isolates, 102 were confirmed as hvKps by the molecular assay, making this method superior to the string test for phenotypic identification of hvKp isolates. To increase the accuracy and sensitivity of hvKp detection from clinical

Page $17 / 22$ 
samples, we also used three key virulence genes as molecular biomarkers previously introduced by Russo et al. We also examined all hvKp isolates for the presence of other virulence factor genes. In general, the frequencies of virulence factor genes, from highest to lowest, iucA, ybt, iutA, rmpA, $k f u$, iroB, peg-344, allS, and magA, respectively, were reported. Other studies have also shown that aerobactin is produced by more than $90 \%$ of hvKp, whereas only $6 \%$ of cKp strains can express it $(16,25)$. In a study by $\mathrm{XU}$ et al. the prevalence of iucA, iutA, rmpA and iro was reported to be $56.8 \%, 56.8 \%, 43.2 \%$ and $40.9 \%$, respectively. The prevalence of iutA and $r m p A$ was similar to our study, but in the present study, the prevalence of iucA was higher and iro was lower than the results of the study by Xu et al (26). The $y b t$ was the second most prevalent virulence factor gene among hvKp isolates in this study. The yersiniabactin gene and its receptor, which is an important virulence factor for the survival of Klebsiella strains under severe conditions, can transmit both an integrative conjugative element (ICEKp) and a plasmid (recently reported) (27). Some studies have described the correlation between yersiniabactin-producing hvKps and pulmonary infectious diseases $(28,29)$. In Iran, a study conducted by Tabrizi et al. Reported that 5 of 53 K.pneumoniae strains isolated from ventilatorassociated pneumonia were hvKp (30). In the current study, of $33 \mathrm{hvKps}$ isolated from lung-related samples, 27 isolates were $y b t-$ positive, confirming the results of previous studies. The $r m p A$ was identified as the fourth most virulence factor. Because $r m p A$ increases the expression of capsular polysaccharide (CPS), we expected that the rmpA-producing hvKp that were isolated would be string test-positive, but this hypothesis was refuted by our results, such that only $36.7 \%$ of the rmpA-positive isolates were reported as hypercystic phenotype. Studies have shown that other genes besides $r m p A$ are involved in capsular gene expression, such as regulation of capsular synthesis $\mathrm{B}(\mathrm{rcs} B)$. Both $r m p A$ and $r c s B$ genes have been shown to co-occur (31). In addition, the data show that the co-presence of four genes (iucA, ybt, iutA, rmpA) was more frequent in hvKp. In addition, other plasmid-born genes such as iro, peg344 were less frequent and were reported only sporadically.

Sequencing and analysis of large virulence plasmids from hvKp strains revealed that virulence-associated genes were mainly found in two regions. The $r m p A 2$, iucABCD and iutA genes are located close to each other, followed by the $r m p A, p e g 344$ and iroBCDN genes in the second region. Some virulence plasmids carry all virulence genes (e.g. pLVPK, accession number: AY378100.), but others have lost one or more virulence-associated loci, confirming our result (e.g. pVir, accession number: CP029383.2) (5, 32). Despite most Asian countries having introduced K1 and K2 as the most common capsular serotypes (3336), we identified K20 as the most common capsular type in Iran. This phenomenon suggests that the prevalence of the different serotypes may vary depending on the geographical area. Although there has been no comprehensive study on the hvKp isolates in Iran and little information is available on them, no K1 and K2 were found among the $K$. pneumoniae isolates in the study conducted by Aghamohammad et al (37). Also, in another study, one K1 and $15 \mathrm{~K} 2$ were identified among 122 K. pneumoniae isolates from Semnan, Iran, which are in agreement with our results (we detected only one K1 and three K2) (38). Another study from Iran conducted by Solgi et al., Reported that the prevalence of K1 and K2 was $45.9 \%$ and $13.5 \%$ respectively, which was more than the present study (39).

Most hvKps are sensitive to most antibiotics except for intrinsic resistance to ampicillin, similarly in this study all hvKp isolates were ampicillin resistant (40). Studies have shown that hvKps are unlikely to take up DNA from other resistant bacteria due to the large size of the capsule and increased expression of capsule-related genes, therefore antibiotic resistance is less common in hvKps than in cKp isolates (41). However, the prevalence of antibiotic-resistant hvKp isolates is increasing worldwide $(42,43)$. The current study revealed the high prevalence of MDR-hvKp and high resistance to imipenem (66\%). Moreover, the presence of bla $a_{\mathrm{TEM}}, b / a_{\mathrm{SHV}}$, bla $a_{\mathrm{CTX}-\mathrm{M} 15}, b / a_{\mathrm{OXA}-48}$ and $b / a_{\mathrm{NDM}-1}$ was detected simultaneously in $56.8 \%$ of hvKp isolates.

Two pathways have been identified for the emergence of MDR-hvKp strains, the horizontal acquisition of resistance genes by plasmids and mobile genetic elements (MGEs) by hvKp isolates (type I), and another pathway is the acquisition of the virulenceassociated plasmid (e.g., pLVPK and pVir) by classical MDR-K. pneumoniae strains (MDR-cKp) (type II) $(42,44)$. Ultimately, both mechanisms lead to the development of MDR-hvKp strains that are resistant to antibiotic treatment in addition to having a very high pathogenicity that poses a serious threat to public health.

The correlation of OmpK36 porin variants with specific sequence types (STs) of K. pneumoniae was first described by Papagiannitsis et al. K. pneumoniae isolates can be classified into four groups (designated groups A to D) by ompK36 genotyping $(12,45)$. There is a relationship between ompK36 type and clonal group (CG). The studies have described the correlation between CG15, CG22, CG23, CG34, CG65 to type A, CG292, CG347, CG1612, CG1928 to group B, CG23, CG48, CG42, 
CG65, CG86, CG76, CG420, CG107 to group C and CG45, CG268, CG420 to group D (46). Some STs were reported to be associated with hvKp isolates, e.g. ST11 and ST23, that ST11 (CG258) belonged to ompK36 group A and ST23 (CG23) belonged to ompK36 group C $(12,46)$. In this study, clonal relatedness by ompK36 typing revealed that group $C(70.6 \%)$ was the most common ompK36 porin type among hvKp isolates. A study in Taiwan showed that ompK36 group $\mathrm{C}$ was significantly more abundant among K. pneumoniae isolates (46). This study was in agreement with our study in Iran.

\section{Conclusion}

This study presented a new screening method based on the resistance of hvKp to tellurite, which was superior to the string test in phenotypic identification of hvKp isolates. A high prevalence of MDR-hvKp and a high level of resistance to imipenem (66\%) were detected. In addition, coexistence of bla $a_{\mathrm{TEM}}, b / a_{\mathrm{SHV}}$, bla $a_{\mathrm{CTX}-\mathrm{M} 15}, b / a_{\mathrm{OXA}-48}$ and $b / a_{\mathrm{NDM}-1}$ was identified in $56.8 \%$ of hvKp isolates. Using the PCR-based ompK36 typing method, which was simpler and less expensive than MLST, we were also able to investigate the clonal relatedness of the strains. It was found that the majority of hvKp isolates belonged to capsular serotype K20 and ompK36 group C, which is related to CG23 (e.g. ST23). It seems that the control of MDR-hvKp is an urgent issue in the healthcare setting.

\section{List Of Abbreviations}

K. pneumoniae, Klebsiella pneumoniae; hvKp, Hypervirulent Klebsiella pneumoniae; rmpA, regulator of mucoid phenotype; magA, mucoviscosity-associated gene; kfu, Klebsiella ferric uptake; iroB, salmochelin iron uptake systems; ybt, yersiniabactin; allS, allantoin metabolism gene; AK, amikacin; GN, gentamicin; CTX, cefotaxime; CAZ, ceftazidime; CRO, ceftriaxone; IMI, imipenem; MRP, meropenem; FEP, cefepime; CIP, ciprofloxacin; AMP, ampicillin; AZM, aztreonam; MIC, Minimum inhibitory concentration; ICU, intensive care unit; ESBL, Extended spectrum beta-lactamases; PLVPK, Large Virulence Plasmid of K. pneumoniae, MDR, multidrug resistant; MDR-cKp, classical MDR-K. pneumoniae, ST, sequence types; Bal, bronchoalveolar lavage; CSF, cerebrospinal fluid.

\section{Declarations}

\section{Acknowledgments}

The authors would like to thank the personnel in the bacteriology department of the Pasture Institute of Iran for their help. This research was supported by the Pasture Institute of Iran.

\section{Conflict of interest}

The authors have no conflicts of interest to declare.

\section{Funding}

The funding source was granted to Rahimeh Sanikhani as Ph.D. student from Pasteur Institute of Iran (project No: B-9427).

\section{Declaration of interests}

The authors declare that they have no known competing financial interests or personal relationships that could have appeared to influence the work reported in this paper.

\section{Competing interests}




\section{Ethical statement}

This project was done based on ethical guidelines as previously approved by the Pasteur Institute of Iran (IR.B-9427).

\section{Authors' contributions}

Hamid Solgi \& Mohammad Moeinirad, collected the samples and their data; Rahimeh Sanikhani, carried out other phenotypic and genotypic tests; Rahimeh Sanikhani \& Mohammad Moeinirad, wrote the manuscript and analyzed the data; Azar Haddadi, Professor of Infectious Diseases; Farzad Badmasti \& Fereshteh Shahcheraghi, supervised the project and write and revised the manuscript.

\section{References}

1. Liu C, Guo J. Hypervirulent Klebsiella pneumoniae (hypermucoviscous and aerobactin positive) infection over 6 years in the elderly in China: antimicrobial resistance patterns, molecular epidemiology and risk factor. Annals of clinical microbiology and antimicrobials. 2019;18(1):4.

2. Rossi B, Gasperini ML, Leflon-Guibout V, Gioanni A, de Lastours V, Rossi G, et al. Hypervirulent Klebsiella pneumoniae in cryptogenic liver abscesses, Paris, France. Emerging infectious diseases. 2018;24(2):221.

3. Dong N, Yang X, Zhang R, Chan EW-C, Chen S. Tracking microevolution events among ST11 carbapenemase-producing hypervirulent Klebsiella pneumoniae outbreak strains. Emerging microbes \& infections. 2018;7(1):1-8.

4. Paterson DL, Siu K, Chang FY. Klebsiella species (K. pneumoniae, K. oxytoca, K. ozaenae and K. rhinoscleromatis). Antimicrobe. 2014;9:74-6.

5. Russo TA, Marr CM. Hypervirulent Klebsiella pneumoniae. Clinical microbiology reviews. 2019;32(3).

6. Xie M, Dong N, Chen K, Yang X, Ye L, Chan EW-C, et al. A hybrid plasmid formed by recombination of a virulence plasmid and a resistance plasmid in Klebsiella pneumoniae. Journal of Global Antimicrobial Resistance. 2020.

7. Liao W, De Wang L, Li D, Du F-I, Long D, Liu Y, et al. High Prevalence of 16s rRNA Methylase Genes Among CarbapenemResistant Hypervirulent Klebsiella pneumoniae Isolates in a Chinese Tertiary Hospital. Microbial Drug Resistance. 2021;27(1):44-52.

8. Wyres KL, Wick RR, Judd LM, Froumine R, Tokolyi A, Gorrie CL, et al. Distinct evolutionary dynamics of horizontal gene transfer in drug resistant and virulent clones of Klebsiella pneumoniae. PLoS genetics. 2019;15(4):e1008114.

9. Feng Y, Lu Y, Yao Z, Zong Z. Carbapenem-resistant hypervirulent Klebsiella pneumoniae of sequence type 36. Antimicrobial agents and chemotherapy. 2018;62(7).

10. Woodford N, Turton JF, Livermore DM. Multiresistant Gram-negative bacteria: the role of high-risk clones in the dissemination of antibiotic resistance. FEMS microbiology reviews. 2011;35(5):736-55.

11. Peirano G, Chen L, Kreiswirth BN, Pitout JD. Emerging antimicrobial-resistant high-risk Klebsiella pneumoniae clones ST307 and ST147. Antimicrobial Agents and Chemotherapy. 2020;64(10).

12. Yan J, Wang M, Zheng P, Tsai L, Wu J. Associations of the major international high-risk resistant clones and virulent clones with specific ompK36 allele groups in Klebsiella pneumoniae in Taiwan. New microbes and new infections. 2015;5:1-4.

13. Hamzaoui Z, Ocampo-Sosa A, Martinez MF, Landolsi S, Ferjani S, Maamar E, et al. Role of association of OmpK35 and OmpK36 alteration and blaESBL and/or blaAmpC genes in conferring carbapenem resistance among non-carbapenemaseproducing Klebsiella pneumoniae. International journal of antimicrobial agents. 2018;52(6):898-905.

14. Yao B, Xiao X, Wang F, Zhou L, Zhang X, Zhang J. Clinical and molecular characteristics of multi-clone carbapenem-resistant hypervirulent (hypermucoviscous) Klebsiella pneumoniae isolates in a tertiary hospital in Beijing, China. International journal of infectious diseases. 2015;37:107-12.

Page 20/22 
15. Peymani A, Farivar TN, Sanikhani R, Javadi A, Najafipour R. Emergence of TEM, SHV, and CTX-M-Extended Spectrum $\beta$ lactamases and class 1 integron among Enterobacter cloacae isolates collected from hospitals of Tehran and Qazvin, Iran. Microbial Drug Resistance. 2014;20(5):424-30.

16. Russo TA, Olson R, Fang C-T, Stoesser N, Miller M, MacDonald U, et al. Identification of biomarkers for differentiation of hypervirulent Klebsiella pneumoniae from classical K. pneumoniae. Journal of clinical microbiology. 2018;56(9).

17. Compain F, Babosan A, Brisse S, Genel N, Audo J, Ailloud F, et al. Multiplex PCR for detection of seven virulence factors and K1/K2 capsular serotypes of Klebsiella pneumoniae. Journal of clinical microbiology. 2014;52(12):4377-80.

18. Yu W-L, Ko W-C, Cheng K-C, Lee C-C, Lai C-C, Chuang Y-C. Comparison of prevalence of virulence factors for Klebsiella pneumoniae liver abscesses between isolates with capsular K1/K2 and non-K1/K2 serotypes. Diagnostic microbiology and infectious disease. 2008;62(1):1-6.

19. Ahmed SF, Ali MMM, Mohamed ZK, Moussa TA, Klena JD. Fecal carriage of extended-spectrum $\beta$-lactamases and AmpCproducing Escherichia coli in a Libyan community. Annals of clinical microbiology and antimicrobials. 2014;13(1):22.

20. Abhari SS, Badmasti F, Modiri L, Aslani MM, Asmar M. Circulation of imipenem-resistant Acinetobacter baumannii ST10, ST2 and ST3 in a university teaching hospital from Tehran, Iran. Journal of medical microbiology. 2019;68(6):860-5.

21. Magiorakos A-P, Srinivasan A, Carey Rt, Carmeli Y, Falagas Mt, Giske Ct, et al. Multidrug-resistant, extensively drug-resistant and pandrug-resistant bacteria: an international expert proposal for interim standard definitions for acquired resistance. Clinical microbiology and infection. 2012;18(3):268-81.

22. Catalán-Nájera JC, Garza-Ramos U, Barrios-Camacho H. Hypervirulence and hypermucoviscosity: two different but complementary Klebsiella spp. phenotypes? Virulence. 2017;8(7):1111-23.

23. Liu C, Guo J. Hypervirulent Klebsiella pneumoniae (hypermucoviscous and aerobactin positive) infection over 6 years in the elderly in China: antimicrobial resistance patterns, molecular epidemiology and risk factor. Annals of clinical microbiology and antimicrobials. 2019;18(1):1-11.

24. Parrott A, Shi J, Aaron J, Green D, Whittier S, Wu F. Detection of multiple hypervirulent Klebsiella pneumoniae strains in a New York City hospital through screening of virulence genes. Clinical Microbiology and Infection. 2021;27(4):583-9.

25. Paczosa MK, Mecsas J. Klebsiella pneumoniae: going on the offense with a strong defense. Microbiology and Molecular Biology Reviews. 2016;80(3):629-61.

26. Xu M, Fu Y, Fang Y, Xu H, Kong H, Liu Y, et al. High prevalence of KPC-2-producing hypervirulent Klebsiella pneumoniae causing meningitis in Eastern China. Infection and drug resistance. 2019;12:641.

27. Lam MM, Wyres KL, Judd LM, Wick RR, Jenney A, Brisse S, et al. Tracking key virulence loci encoding aerobactin and salmochelin siderophore synthesis in Klebsiella pneumoniae. Genome medicine. 2018;10(1):1-15.

28. Bachman MA, Oyler JE, Burns SH, Caza M, Lépine F, Dozois CM, et al. Klebsiella pneumoniae yersiniabactin promotes respiratory tract infection through evasion of lipocalin 2. Infection and immunity. 2011;79(8):3309-16.

29. Lawlor MS, O'connor C, Miller VL. Yersiniabactin is a virulence factor for Klebsiella pneumoniae during pulmonary infection. Infection and immunity. 2007;75(3):1463-72.

30. Tabrizi AMA, Badmasti F, Shahcheraghi F, Azizi O. Outbreak of hypervirulent Klebsiella pneumoniae harbouring blaVIM-2 among mechanically-ventilated drug-poisoning patients with high mortality rate in Iran. Journal of global antimicrobial resistance. 2018;15:93-8.

31. Cheng H, Chen Y, Wu C, Chang H, Lai Y, Peng H-L. RmpA regulation of capsular polysaccharide biosynthesis in Klebsiella pneumoniae CG43. Journal of bacteriology. 2010;192(12):3144-58.

32. Struve C, Roe CC, Stegger M, Stahlhut SG, Hansen DS, Engelthaler DM, et al. Mapping the evolution of hypervirulent Klebsiella pneumoniae. MBio. 2015;6(4).

33. Fang C-T, Lai S-Y, Yi W-C, Hsueh P-R, Liu K-L, Chang S-C. Klebsiella pneumoniae genotype K1: an emerging pathogen that causes septic ocular or central nervous system complications from pyogenic liver abscess. Clinical infectious diseases. 2007;45(3):284-93.

34. Victor LY, Hansen DS, Ko WC, Sagnimeni A, Klugman KP, Von Gottberg A, et al. Virulence characteristics of Klebsiella and clinical manifestations of K. pneumoniae bloodstream infections. Emerging infectious diseases. 2007;13(7):986.

Page 21/22 
35. Liu YM, Li BB, Zhang YY, Zhang W, Shen H, Li H, et al. Clinical and molecular characteristics of emerging hypervirulent Klebsiella pneumoniae bloodstream infections in mainland China. Antimicrobial agents and chemotherapy.

2014;58(9):5379-85.

36. Yeh K-M, Kurup A, Siu L, Koh Y, Fung C-P, Lin J-C, et al. Capsular serotype K1 or K2, rather than magA and rmpA, is a major virulence determinant for Klebsiella pneumoniae liver abscess in Singapore and Taiwan. Journal of clinical microbiology. 2007;45(2):466-71.

37. Aghamohammad S, Badmasti F, Solgi H, Aminzadeh Z, Khodabandelo Z, Shahcheraghi F. First report of extended-spectrum betalactamase-producing Klebsiella pneumoniae among fecal carriage in Iran: high diversity of clonal relatedness and virulence factor profiles. Microbial Drug Resistance. 2020;26(3):261-9.

38. Pajand O, Darabi N, Arab M, Ghorbani R, Bameri Z, Ebrahimi A, et al. The emergence of the hypervirulent Klebsiella pneumoniae (hvKp) strains among circulating clonal complex 147 (CC147) harbouring bla NDM/OXA-48 carbapenemases in a tertiary care center of Iran. Annals of clinical microbiology and antimicrobials. 2020;19:1-9.

39. Solgi H, Shahcheraghi F, Bolourchi N, Ahmadi A. Molecular characterization of carbapenem-resistant serotype K1 hypervirulent Klebsiella pneumoniae ST11 harbouring blaNDM-1 and blaOXA-48 carbapenemases in Iran. Microbial Pathogenesis. 2020;149:104507.

40. Tang M, Kong X, Hao J, Liu J. Epidemiological characteristics and formation mechanisms of multidrug-resistant hypervirulent Klebsiella pneumoniae. Frontiers in Microbiology. 2020;11.

41. Zhu J, Wang T, Chen L, Du H. Virulence factors in hypervirulent Klebsiella pneumoniae. Frontiers in Microbiology. 2021;12:734.

42. Gu D, Dong N, Zheng Z, Lin D, Huang M, Wang L, et al. A fatal outbreak of ST11 carbapenem-resistant hypervirulent Klebsiella pneumoniae in a Chinese hospital: a molecular epidemiological study. The Lancet infectious diseases. 2018;18(1):37-46.

43. Li W, Sun G, Yu Y, Li N, Chen M, Jin R, et al. Increasing occurrence of antimicrobial-resistant hypervirulent (hypermucoviscous) Klebsiella pneumoniae isolates in China. Clinical infectious diseases. 2014;58(2):225-32.

44. Liu Y, Long D, Xiang T-X, Du F-L, Wei DD, Wan L-G, et al. Whole genome assembly and functional portrait of hypervirulent extensively drug-resistant NDM-1 and KPC-2 co-producing Klebsiella pneumoniae of capsular serotype K2 and ST86. Journal of Antimicrobial Chemotherapy. 2019;74(5):1233-40.

45. Papagiannitsis CC, Giakkoupi P, Kotsakis SD, Tzelepi E, Tzouvelekis LS, Vatopoulos AC, et al. OmpK35 and OmpK36 porin variants associated with specific sequence types of Klebsiella pneumoniae. Journal of chemotherapy. 2013;25(4):250-4.

46. Yan J-J, Zheng P-X, Wang M-C, Tsai S-H, Wang L-R, Wu J-J. Allocation of Klebsiella pneumoniae bloodstream isolates into four distinct groups by ompK36 typing in a Taiwanese university hospital. Journal of clinical microbiology.

2015;53(10):3256-63. 